OPEN ACCESS

Edited by:

Catherine Dendrinou-Samara, Aristotle University of

Thessaloniki, Greece

Reviewed by: Guangchao Zheng,

Zhengzhou University, China Paula C. Angelomé Consejo Nacional de Investigaciones Científicas y Técnicas (CONICET), Argentina

*Correspondence:

Akshaya K. Samal s.akshaya@jainuniversity.ac.in

Specialty section: This article was submitted to Nanoscience, a section of the journa

Frontiers in Chemistry

Received: 18 December 2019 Accepted: 07 April 2020

Published: 19 May 2020

Citation:

Bhol P, Bhavya MB, Swain S, Saxena M and Samal AK (2020)

Modern Chemical Routes for the Controlled Synthesis of Anisotropic Bimetallic Nanostructures and Their

Application in Catalysis

Front. Chem. 8:357.

doi: 10.3389/fchem.2020.00357

\section{Modern Chemical Routes for the Controlled Synthesis of Anisotropic Bimetallic Nanostructures and Their Application in Catalysis}

\author{
Prangya Bhol, M. B. Bhavya, Swarnalata Swain, Manav Saxena and Akshaya K. Samal* \\ Centre for Nano and Material Sciences, Jain Global Campus, Jain University, Ramanagara, India
}

Bimetallic nanoparticles (BNPs) have attracted greater attention compared to its monometallic counterpart because of their chemical/physical properties. The BNPs have a wide range of applications in the fields of health, energy, water, and environment. These properties could be tuned with a number of parameters such as compositions of the bimetallic systems, their preparation method, and morphology. Monodisperse and anisotropic BNPs have gained considerable interest and numerous efforts have been made for the controlled synthesis of bimetallic nanostructures (BNS) of different sizes and shapes. This review offers a brief summary of the various synthetic routes adopted for the synthesis of Palladium(Pd), Platinum(Pt), Nickel(Ni), Gold(Au), Silver(Ag), Iron(Fe), Cobalt(Co), Rhodium(Rh), and Copper(Cu) based transition metal bimetallic anisotropic nanostructures, growth mechanisms e.g., seed mediated co-reduction, hydrothermal, galvanic replacement reactions, and antigalvanic reaction, and their application in the field of catalysis. The effect of surfactant, reducing agent, metal precursors ratio, $\mathrm{pH}$, and reaction temperature for the synthesis of anisotropic nanostructures has been explained with examples. This review further discusses how slight modifications in one of the parameters could alter the growth mechanism, resulting in different anisotropic nanostructures which highly influence the catalytic activity. The progress or modification implied in the synthesis techniques within recent years is focused on in this article. Furthermore, this article discussed the improved activity, stability, and catalytic performance of BNS compared to the monometallic performance. The synthetic strategies reported here established a deeper understanding of the mechanisms and development of sophisticated and controlled BNS for widespread application.

Keywords: anisotropic nanostructures, galvanic reaction, antigalvanic reaction, bimetallic nanostructures, catalysis

\section{INTRODUCTION}

Bimetallic nanostructures (BNS) have gained worldwide attention due to the enhanced properties they have compared to its counterparts and have widely been explored in different applications (Sharma et al., 2017). In the early 1980s the words "bimetallic cluster" were first introduced by John H. Sinfelt during his series of research over bimetallic nanocatalysts supported on silica or alumina (Sinfelt et al., 1980, 1981, 1982). BNS were synthesized by combining two different metals 
where the constituent metals either form an alloy or a nanocomposite including core shell and nanodendrites (Tao, 2012). The presence of two or more metals with their respective nanometric shape and size decides the development of novel properties from its pristine form. Moreover, the formation of self-accumulation structures has been gaining significant interest and suggests attractive options for the synthesis of bimetallic nanomaterials with unique functions (Rechberger and Niederberger, 2017). Unique properties developed in a single material from the manifestation of multiple metals can be used in different applications such as catalysis, sensing, thermoelectric, and electronic devices (Barakat et al., 2010; Alonso et al., 2012; Piccolo, 2012; Tao, 2012; Liu and Astruc, 2017). Diverse routes have been adapted for the synthesis of bimetallic nanoparticles, regardless of the engineering of these bimetallic structures with monodispersity. Controlled shape and size in bulk is the most important challenge.

Why are anisotropic nanomaterials with monodispersity in incessant demand? When anisotropy is introduced to any nanoparticle for various shapes, sizes, and monodisperse morphologies (such as rods, cubes, octahedrons, dodecahedrons, triangles, etc.) different facets with different surface energies are generated, producing novel physical and chemical properties (Sajanlal et al., 2011). In addition to that, two or more metals forming anisotropic nanostructures such as core-shells, alloys, or complexes alter the atomic and electronic arrangements in them and attribute better characteristic performance (Alonso et al., 2012; Tao, 2012; Ruditskiy et al., 2016; Tao et al., 2016). Moreover, the shape of the nanomaterial also affects the motion of electrons, holes, plasmons, and excitons according to its directional growth and hence generates different properties. For example, in the case of an isotropic nanoparticle (i.e., a sphere) the electrons are confined symmetrically in all directions and, hence, the properties will be more or less same in all direction. Whereas in the case of anisotropic nanomaterials, which are further classified as 1D, 2D, and 3D nanostructures, further directional dependent physical and chemical properties are posed because of the available motion of electron in these dimensions (Sajanlal et al., 2011). The facets possess a disproportionate number of atoms or molecules over the surface which influence the reactivity, catalytic activity, resistance, and optoelectronics properties. Different facets play a considerable role in enhancing the catalytic activity or efficiency because the atoms present in different planes, edges, or corners possess different surface energies and thus different reactivities (Mahmoud et al., 2013; Mahmoud and El-Sayed, 2014). The sharp edge present in the corner shows improved reactivity in comparison to the atoms present in other sites or in isotropic particles (Tao et al., 2008). Further alteration of the elemental composition in the synthesis of complex nanocatalysts opens up new dimensions due to the development of new facets and electronic properties. Enhanced properties are achieved from bimetallic nanocatalysts than from its monometallic counterparts (Wang et al., 2011). BNS have been widely explored in the combination of rare elements such as $\mathrm{Au}, \mathrm{Pd}$, and $\mathrm{Pt}$ with cheaper metals like $\mathrm{Ni}, \mathrm{Cu}$, and $\mathrm{Fe}$ for enhanced catalytic activity, selectivity, high recovery, and large-scale industrial applications due to its development of novel characteristics.
Additionally, bimetallic nanocatalysts have shown effective catalytic behavior and an enriched stability and activity compared to monometallic NPs (Mahmoud and El-Sayed, 2014). BNPs have been synthesized in various shapes, sizes, and structures using different protocols (Yuan et al., 2015). The reaction environment plays a key role in establishing the structure and property of the multiple metals participating in the reaction (Santana et al., 2018). The general method to synthesize bimetallic nanostructure involves the successive reduction of metal ions providing an appropriate stabilizing agent in order to avoid agglomeration as well as preferential growth of the nanostructure. The traditional synthesis method for bimetallic nanostructure uses template and surfactant processes (Ahmad et al., 2019), sol-gel methods (Gołabiewska et al., 2017), and electrochemical depositions (Vilana et al., 2015). These methods require the removal of the template, surfactant, and substrate for the purification of product. Moreover, certain reactions have been conducted at elevated temperatures and have resulted in large unalloyed NPs, lowered the probability of any interaction of the two metals, and provided their respective characteristics (Mallikarjuna et al., 2018). To overcome these drawbacks, many advanced or modified methods have been proposed for the synthesis of BNS where the interaction of two metals provides high stability, enhanced activity, and unique electrical and chemical properties. The recent discovery of an antigalvanic reaction (AGR) which contradicts the galvanic principle has attracted much attention for its synthesis, mechanism, and catalytic applications (Gan et al., 2018). Transition metal NPs such as $\mathrm{Pd}, \mathrm{Pt}, \mathrm{Ni}$, and $\mathrm{Cu}$ have been widely used due to the easy synthetic protocol and have a wide range of applications in the field of catalysis.

Here, we have discussed most of the remarkable anisotropic BNS such as cubic nanoframes (Ye et al., 2015), nanoboxes (Ye et al., 2015), nanocubes (Lim et al., 2010), nanorods (Sun et al., 2017), octopods (Kunz et al., 2017), and trigonal nanoframes (Chen et al., 2015) synthesized by different techniques such as seed mediated method (Kunz et al., 2017), galvanic method (González et al., 2011), hydrothermal method (Wu et al., 2019b), and electrospinning technique (Ghouri et al., 2015). The major objective of this article is to discuss the updated synthesis mechanisms as well as to provide a better understanding of the factors responsible for generating complex bimetallic anisotropic nanostructures with high monodispersity. In addition to that, the discussion highlights the importance of bimetallic properties in the field of catalysis, photocatalysis, and electrocatalysis. Highly dispersed anisotropic BNS offers a fascinating path to design new catalysts with novel properties and reactivity with respect to different facets. In order to understand the concept, the discussion starts gradually with the history and the revolution in the synthetic approaches to achieve monodispersed anisotropic bimetallic nanostructures. The discussion is further exemplified with a few illustrations of different techniques giving information about factors such as precursor's ratio, reaction $\mathrm{pH}$, temperature, and capping agents responsible in engineering the final morphology and internal composition. The review further explicitly highlights galvanic replacement reactions mechanisms and its uses to develop complex BNS. New approaches adapted through a combination with other mechanisms like Kirkendall, 
sequential deposition, and co-reduction have been explained with examples.

\section{Revolution of Synthetic Methods}

A significant amount of knowledge has been gathered on the synthetic approaches of BNS over the last few decades. The general strategy to synthesize nanomaterial is either "top-down methods" or "bottom-up methods" as shown in Figure 1 (Ovais et al., 2017). The top-down method starts with a bulk structure and then it reduces to nanoscale via an etching out of the crystal planes of the initial structure. In a general way it could be said that the building blocks are removed from the bulk to shape it into desired nanostructures. This process involves physical processes such as the mechanical grinding or ball milling methods that grins the bulk material into a fine powder with the selective addition of stabilizing agents to prevent agglomeration and result in a nanomaterial (Yadav et al., 2012). Although these mechanical methods provide the least expensive paths to synthesize nanomaterials, where kinetic energy was developed during the breaking up of the bulk structure is useful in the reduction of materials. This method fails to synthesize fine anisotropic nanostructures with high monodispersity.

The breaking of bulk often results in uneven surfaces or ruptured edges in the nanomaterial which can further impact on the physiochemical property as well as the surface energy of the BNS. Bottom up method resolves these issues and provides a fascinating path to designing monodispersed anisotropic nanostructures. In this method, the nanostructures are assembled from the bottom by stacking atom-by-atom, molecule-bymolecule resulting in a uniform distribution of building blocks. The method starts from the reduction of precursors to atoms followed by nucleation and growth process.

In the year 1857, Michael Faraday was the first person to propose the general method of chemical reduction of gold and other metal salts in the presence of a suitable stabilizer to synthesize a metal nanoparticle dispersed in an aqueous solvent, which turned out to be the most powerful and common technique in the synthesis field (Faraday, 1857). After his discovery, Turkevich carried out several experiments and established a standard synthesis protocol where he successfully synthesized Pt (Aika et al., 1976), Au (Turkevich et al., 1951) and Pd (Turkevich and Kim, 1970) nanoparticles using sodium citrate as the reducing agent for the supported catalyst. He concluded that the mechanism involves successive nucleation, growth, and agglomeration forming nanoparticles. Hence, the rate of nucleation and growth of the cluster determines the size of the particle. With passing time, the research into bimetallic nanoparticle generated great interest. A series of studies carried out on bimetallic nanoparticles as catalysts over silica such as $\mathrm{Ru}-\mathrm{Cu}$ (Sinfelt et al., 1980), Os-Cu (Sinfelt et al., 1981), and Pt-Ir (Sinfelt et al., 1982) by Sinfelt et al. over bimetallic nanostructures influencing the catalytic activity. The morphology of bimetallic nanostructures was analyzed using $\mathrm{X}$-ray absorption fine structure (EXAFS) technique and confirmed the spherical structure of BNPs with homogeneous distribution of two metals forming an alloy (Meitzner et al., 1983, 1985). BNPs synthesis involves either co-reduction or successive reduction of two metal precursors. The co-reduction method is the simplest approach to synthesizing bimetallic nanoparticles and is similar to monometallic nanoparticle synthesis, where successive reduction of two metals is followed in order to synthesize core-shell bimetallic nanostructure. The efforts of countless researchers have made tight control over the morphology and composition of BNS possible.

Bimetallic nanoparticles such as $\mathrm{Au}-\mathrm{Pt}$ or $\mathrm{Pd}-\mathrm{Pt}$ are synthesized using sodium citrate as a reducing agent (Harada et al., 1992). Polymer-stabilized bimetallic nanoparticles in a water-alcohol system was also studied (Toshima and Wang, 1993, 1994a,b; Schmidt et al., 1997). The synthesis of Pd-Pt bimetallic nanoparticles under the simultaneous reduction of two metal precursors i.e. palladium (II) chloride and hexachloroplatinic (IV) acid was carried out using a stabilizing agent, poly(N-vinyl-2-pyrrolidone) (PVP) in continuous reflux of ethanol-water $(1: 1 \mathrm{v} / \mathrm{v})$. The synthesis of light transition metals nanoparticles is difficult because of its lower redox potentials. But light transition metal nanoparticles are very important in the field of catalysis and as magnetic materials. These are prepared with the modified alcohol reduction method with additional treatment of several other chemical components like alcohol, glucose, $\mathrm{NaOH}$, and tetraoctylammoniumbromide with a polymer which influences the synthesis of bimetallic nanoparticles.

Thermal decomposition in the presence of PVP was reported by Bradley with a certain modification of method (Esumi et al., 1990; Bradley et al., 1993). In the reaction, heating of palladium and copper acetates in the presence of a high boiling point solvent, ethoxyethanol, produces a PVP-stabilized $\mathrm{Pd}-\mathrm{Cu}$ nanoparticle. Surfactant assisted bimetallic nanoparticles of gold/palladium using ultrasonic irradiation was reported by Mizukoshi and co, which later was termed the sonochemical method (Mizukoshi et al., 1997). In the synthesis, the surfactant, sodium dodecyl sulfate (SDS), was used to stabilize the reaction where constant ultrasonic irradiation reduces the $\mathrm{Au}(\mathrm{III})$ and $\operatorname{Pd}(\mathrm{II})$ ions in the aqueous medium. Radiolysis synthesis is another highly exploited synthetic method from the 1990s where numerous reports confirmed the synthesis of bimetallic by $\gamma$ radiolysis. The method was mostly used to synthesize bimetallic of noble metals [for example, Ag-Pt BNPs prepared by radiolysis of the aqueous mixture of $\mathrm{Ag}_{2} \mathrm{SO}_{4}$ and $\mathrm{K}_{2} \mathrm{PtCl}_{4}$ (Remita et al., 1996)]. Similarly, $\gamma$ radiolysis was used to synthesize Ag-Au bimetallic nanoparticles with an Au layer over an Ag nanoparticle (Henglein, 1993). Most of the techniques discussed above include the early efforts of researchers in order to design reproducible methods for synthesizing BNPs with the reduction of metal ions in the aqueous medium with suitable treatment of a stabilizing agent.

With the help of reducing agents such as trisodium citrate, hydrazine hydrate, ascorbic acid, alcohols, carbon monoxide, $\mathrm{LiAlH}_{4}$, and $\mathrm{NaBH}_{4}$, the metal ions are reduced to their nanometric size and shape (Service, 2007; Armbrster et al., 2010). The evolution of each technique was accomplished with modifications in the past reports and focused on the monodispersed controlled synthesis of bimetallic nanoparticles. The successful synthesis of BNS encourages researchers to synthesize more complex multimetallic nanostructures. Selective 


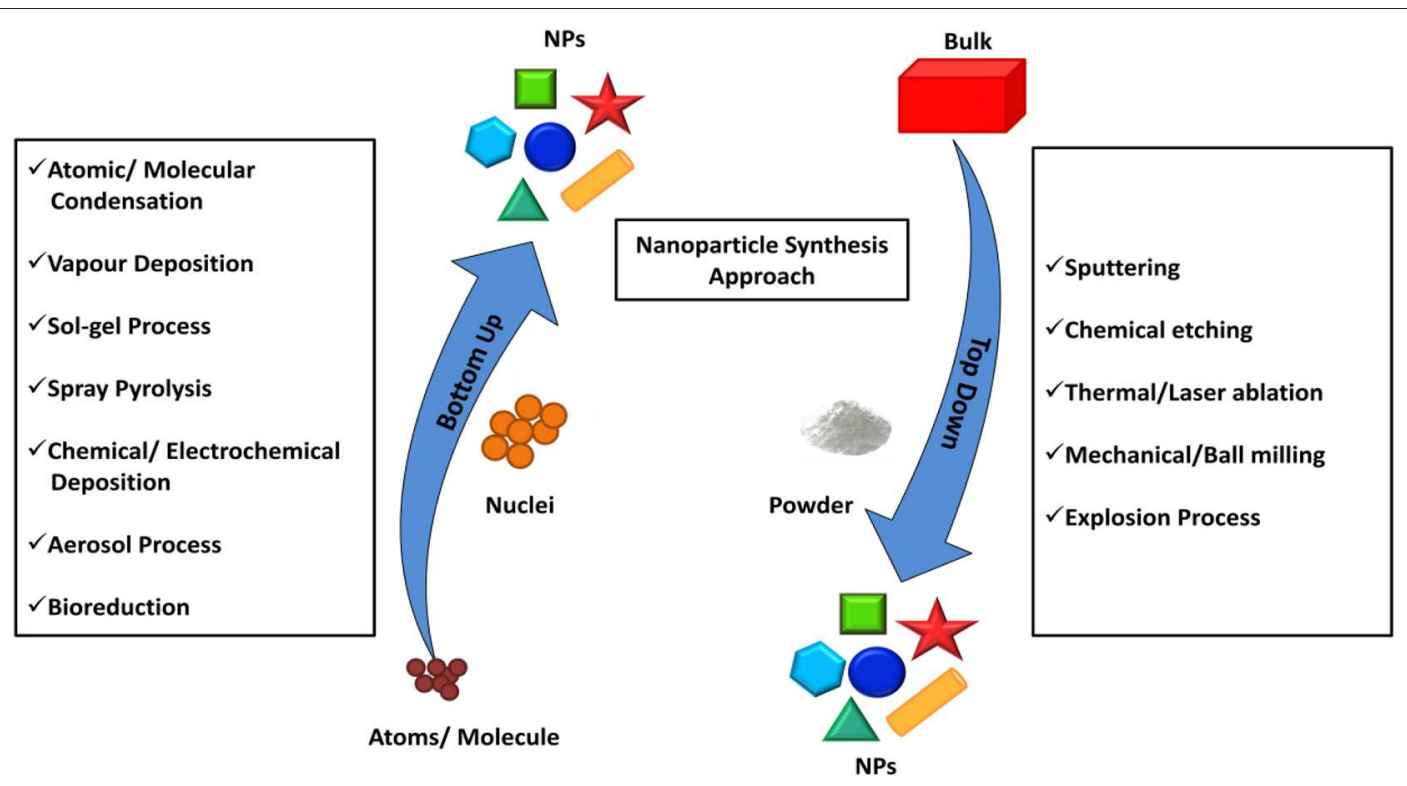

FIGURE 1 | Top-down and bottom-up approaches exploiting different physical, chemical, and biological methods for the synthesis of NPs. Redrawn from the data in Ovais et al. (2017).

element segregation, diffusion, and electrochemical etching of metals have been employed to synthesize desired multimetallic nanostructures (Saleem et al., 2019). The monodispersity in the nanostructure offers facet dependence physiochemical properties which can highly influence the catalytic performance. Several attempts were done by adapting control reaction conditions to get fine monodispersity in the nanostructures.

\section{SYNTHETIC METHODS}

\section{Seed Mediated Growth Technique}

Seed mediated growth mechanism is one of the most common and traditional techniques for the synthesis of anisotropic bimetallic nanostructures. As bimetallic nanostructures involve two metal precursors, the first step is the reduction of one metal using a preferential choice of reducing agent and surfactant to form seeds. Then, the other metal precursor is added in the presence of the seed with surfactant and reducing agent for the controlled overgrowth of the second metal on the well-defined surface of the seeds of the first metals. Physical parameters have played a major role in the structural growth of the BNS. Lattice match, correlation of surface interfacial energies, electro negativities between the two metals, and reduction potentials are the crucial factors which are responsible for the nucleation and growth of bimetallic nanocrystals (Mahmoud and El-Sayed, 2014). Reducing and capping agents have also been considered as vital factors that govern the growth of nanostructures. The reduction potential of the two metals in bimetallic determine the formation of an alloy or core-shell. Metals having very close reduction potentials follow the seed mediated co-reduction (SMCR) technique and reduce together to form an alloy. If two metals have a significant difference in their reduction potentials, they form a preferentially core-shell structure. Pd-Au bimetallic nanocubes ( $\mathrm{Lim}$ et al., 2010), Pd-Cu diverse nanostructures (Kunz et al., 2017), Au-Pd bimetallic nanorods (Sun et al., 2017), and Au-Pd nano rings (Wang W. et al., 2016) were some examples synthesized using the seed mediated method.

\section{Nanocubes}

$\mathrm{Au}$ and $\mathrm{Pd}$ have been investigated more extensively compared to other BNS owing to their excellent catalytic performance compared to their monometallic counterparts. Both Au and Pd have a face-centered cubic (fcc) crystalline arrangement with slight mismatch $(\sim 4 \%)$ and can form materials with a wide range of stoichiometry which provides a path to designing diverse $\mathrm{Au}-\mathrm{Pd}$ nanostructures. Regardless of differences in their redox potential they may reduce together to form alloys via controlling the diffusion environment where the surface deposition occurs faster than diffusion of reactants to the surface of nucleus. PdAu core-shell nanocrystals are synthesized using seed mediated method reported elsewhere (Lim et al., 2010). The thickness of the $\mathrm{Au}$ shell depends on the concentration of a gold (III) chloride $\left(\mathrm{HAuCl}_{4}\right)$ precursor. Initially, $\mathrm{Pd}$ nanocube seed was synthesized using L-ascorbic acid (AA) and bromide ions as the reducing and capping agents, respectively, and favors the growth in (100) direction. Further, the accumulation of $\mathrm{Au}$ on the surface of cubic Pd seeds by the reduction of $\mathrm{HAuCl}_{4}$ in presence of mild reducing agent, AA was carried out. Different concentrations of $\mathrm{HAuCl}_{4}$ were added to $\mathrm{Pd}$ nanocubes for the controlled overgrowth of different thicknesses of $\mathrm{Au}$ on $\mathrm{Pd}$ as shown in Figures 2A-C. TEM image confirmed the overgrowth of $\mathrm{Au}$ as a shell of thickness $1-2 \mathrm{~nm}$ on the Pd nanocubes. The shape of the Pd core retained its original cubic shape, indicating that there is no loss of Pd during the accumulation of an Au shell.

The shell thickness of $\mathrm{Au}$ on $\mathrm{Pd}$ is dependent on different concentrations of $\mathrm{HAuCl}_{4}$, forming a core-shell. Upon increasing 

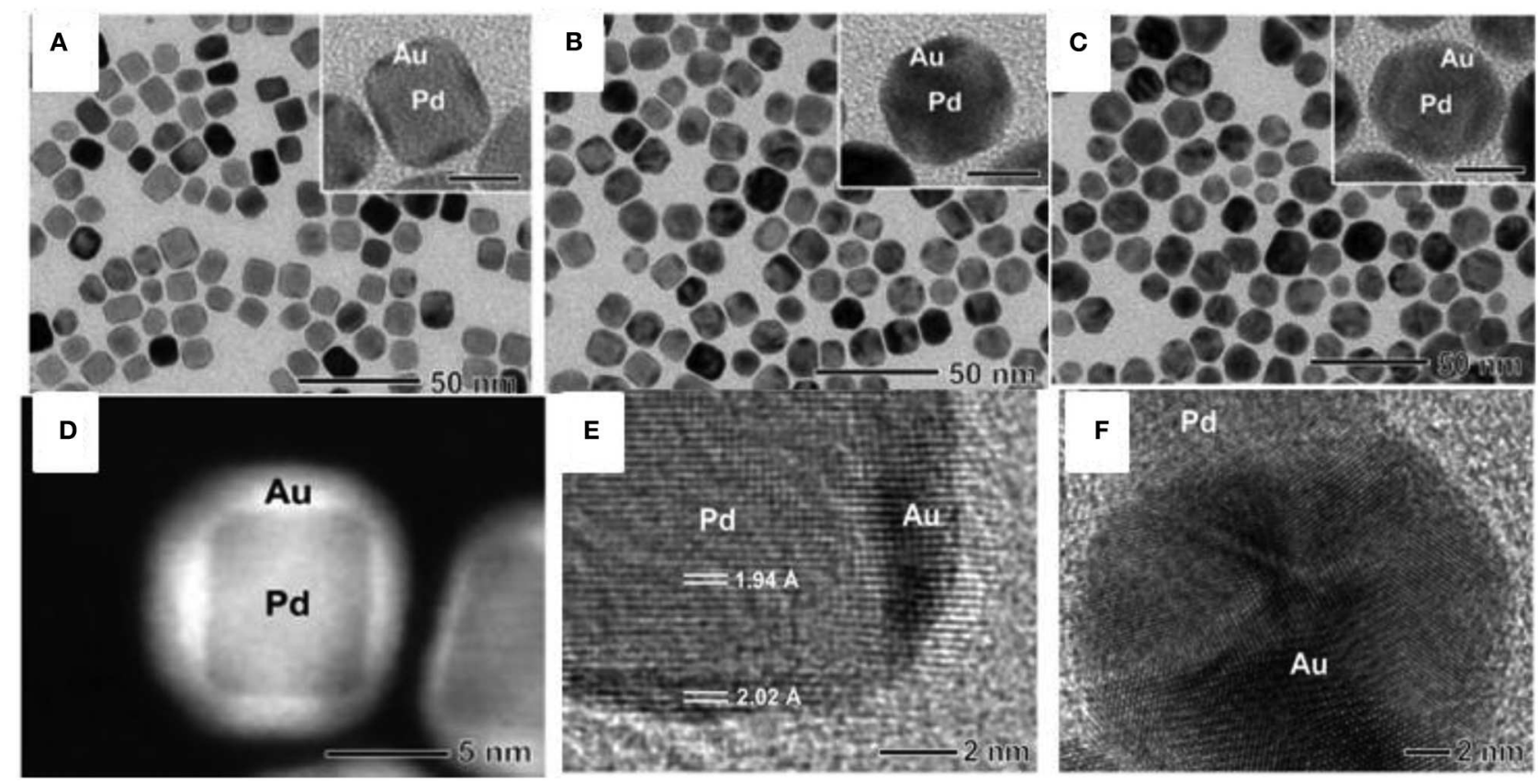

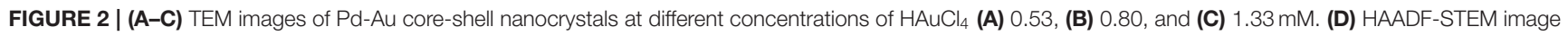
of Pd-Au core-shell nanocrystals. (E) HRTEM image of one of the Pd-Au core-shell nanocrystals. (F) HRTEM image of a Pd-Au dimer, showing the partial decahedral structure of an Au particle attached to a Pd nanocube [Reprinted with permission from Lim et al. (2010), Copyright 2010, American Chemical Society].

the concentration of $\mathrm{HAuCl}_{4}$, the thickness of Au shells increased 3-5 $\mathrm{nm}$ accordingly on Pd cubes without altering the shape of the Pd core as shown in Figure 2A. A bimetallic core-shell nanostructure was clearly seen in a high-angle annular dark-field scanning transmission electron microscopy (HAADF-STEM) image as shown in Figure 2D. This can be seen due to the contrast between the Pd and Au metals. At a low concentration of $\mathrm{HAuCl}_{4}$, the thinner shell of $\mathrm{Au}$ coated over all facets of the Pd nanocube. The high-resolution transmission electron microscopy (HRTEM) image in Figure 2E shows the inter planar distance of $\mathrm{Pd}$ and $\mathrm{Au}$ indicated an epitaxial growth of $\mathrm{Au}$ on $\mathrm{Pd}$. Figure 2F shows the high resolution TEM image of $\mathrm{Pd}-\mathrm{Au}$ dimer, the Au develops with partial decahedral structure over Pd nanocube.

\section{Nanooctopods}

The seed mediated co-reduction (SMCR) technique is used to synthesize $\mathrm{Pd}-\mathrm{Cu}$ nanostructures of larger lattice mismatch $(\sim 7 \%)$ between precursor metals (Kunz et al., 2017). In this technique, a variety of alloy structures are synthesized due to the co-deposition of Pd and $\mathrm{Cu}$ on top of cubic Pd seeds. Pd nanocube seed was prepared by mixing a Pd precursor with cetyltrimethylammonium bromide $(\mathrm{CTAB})$ as a capping agent and $\mathrm{AA}$ as a reducing agent. A calculated amount of seed particles were added to the solution containing metals precursors, $\mathrm{H}_{2} \mathrm{PdCl}_{4}$ and $\mathrm{CuCl}_{2}$, with $\mathrm{AA}$ and $\mathrm{CTAB}$ for the growth of the alloy. CTAB acts not only as a capping agent but also slows the reduction process of the $\mathrm{Pd}$ precursor through its coordination with Pd (II) and forms a [CTA $]_{2} \mathrm{PdBr}_{4-\mathrm{x}} \mathrm{Cl}_{\mathrm{x}}$ complex. Diverse bimetallic $\mathrm{Pd}-\mathrm{Cu}$ nanostructures are synthesized by altering the $\mathrm{Pd}$ and $\mathrm{Cu}$ precursor ratio as well as the $\mathrm{pH}$ of the reaction.
The concentration of copper was kept constant whereas the concentration of the Pd precursor was increased and the ratio of Pd: $\mathrm{Cu}$ maintained from 1:10 to 3:2. There was a gradual increase in the size due to enough availability of the Pd precursor for deposition.

Reaction $\mathrm{pH}$ was monitored by varying $\mathrm{HCl}$ concentrations in the reaction. When the reaction $\mathrm{pH}$ was less $(<2)$, polyhedral structure was favored, while a gradual increase in the reaction $\mathrm{pH}(2-3)$ saw the formation of multi-branched and octopodal structures. This happened because at a lower $\mathrm{pH}$, the slow reduction rate allows the metal atoms to diffuse to a lower energy site throughout the growth, forming a polyhedral structure. The cubic Pd remained at the center and acted as seed for the growth of $\mathrm{Cu}$. The metal with a higher concentration remained in the core part of the structure and the metals with less concentration shifted to the tips of the nanostructures as a shell.

\section{Nanorods}

Au-Pd bimetallic nanorods were prepared by the co-reduction of $\mathrm{Au}$ and $\mathrm{Pd}$ using the seed-mediated method where the growth was restricted kinetically (Sun et al., 2017). First, single crystalline seeds of $\mathrm{Au}$ nanorods were synthesized by mixing $0.5 \mathrm{mM} \mathrm{HAuCl}_{4}$ with $0.2 \mathrm{M} \mathrm{CTAB}$ in (1:1) volume ratio. Then, freshly prepared $0.6 \mathrm{~mL}(0.01 \mathrm{M}) \mathrm{NaBH}_{4}$ in water was injected into the prepared $\mathrm{Au}$ (III)-CTAB mixture with continuous stirring at 12,000 rpm. The color of solution altered from yellow to brownish yellow. Then, the seed solution was allowed to grow for $30 \mathrm{~min}$ (Ye et al., 2013). After the synthesis of single crystalline seeds of $\mathrm{Au}$ nanorods, multifaceted $\mathrm{Au}$ Pd bimetallic alloy shells were overgrown via seed mediated electroless plating. The electroless plating was conducted by 
mixing metal precursors, $\mathrm{HAuCl}_{4}$ and $\mathrm{H}_{2} \mathrm{PdCl}_{4}$, with $\mathrm{CTAB}$ and $\mathrm{AA}$ at $30^{\circ} \mathrm{C}$ under ambient air. Since $\mathrm{Au}$ nanorods hold diverse facets, including local high-index and low-index facets, these can be tuned with different parameters to engineer into diverse architect with the overgrowth of the second metal in the growth step. Metal precursors ratio, reducing agent concentration, and the capping surfactants are responsible for the thickness dependent growth and surface co-deposition. These parameters also influenced atomic coordination and the stoichiometry maintained in the compositional form, resulting in different polyhedral nanostructures. For example, varying $\mathrm{CTAB}$ concentrations alters packing densities on different facets which favors growth in different geometries in BNS. CTAB forms bilayers on the surface of Au nanorods and regulates the diffusion rate and co-deposition ensuing alloy of Au-Pd. Surface growth of metals on Au nanorods leads to the overgrowth of nanocrystals and transforms into structurally distinct multifaceted geometries of $\mathrm{Au}$ and $\mathrm{Pd}$ alloy and core-shell nanorods. The electroless deposition of Au-Pd alloy over the Au nanorod seed was manipulated kinetically, which possibly resulted in huge families of polyhedral Au-Pd bimetallic nanorods with fine geometries including elongated hexoctahedral, truncated concave cuboidal, and truncated cuboidal nanorods.

\section{Nanorings}

Nanocrystals with hollow interiors have attracted great interest as they contain a larger surface-to-volume ratio in comparison to the solid counterpart which crucially affects the performance in different applications (Banaee and Crozier, 2010). Au nanorings are active materials and show significant tunability in localized surface plasmon resonance (LSPR) properties connected to variable transverse and longitudinal axes (Ozel et al., 2015). Wang et al. studied a seed-mediated route for the synthesis of Pd nanosheets supported by Au nanorings (Wang W. et al., 2016). The island growth of Au NPs due to the selective deposition on the sidelines of Pd nanosheets formed Pd ultrathin nanosheet supported by $\mathrm{Au}$ nanorings. Pure $\mathrm{Au}$ rings were further synthesized by selectively removing Pd cores by etching the Pd with excess nitric acid $\left(\mathrm{HNO}_{3}\right)$ at room temperature. The expansion of $\mathrm{Au}$ rings over the Pd nanosheets was monitored in the ultraviolet-visible (UV-Vis) spectroscopy. The nanosheets formed from Pd showed a large LSPR band around $\sim 940 \mathrm{~nm}$. As the Au NPs slowly grown on the Pd nanosheets surface were covered completely, Pd peak disappeared due to the plasmon interaction between the Au and Pd nanosheets and two distinct peaks appeared at 520 and $770-915 \mathrm{~nm}$. The peak appeared at $520 \mathrm{~nm}$ was due to the out of plane dipole mode of the Au rings and peak in the near infrared region was due to the combination of the in-plane dipole and face resonance modes in the nanorings (Ozel et al., 2015).

\section{Nanobipyramids}

Anisotropic $\mathrm{Au} / \mathrm{SiO}_{2} / \mathrm{Pd}$ nanobipyramids (NBPs) were successfully synthesized through the seed-mediated growth mechanism with a silica coating, which acts as a hard template to provide a preferential deposition of Pd over exposed surfaces of Au NBPs. The first step was the formation of Au NBPs seed by the reduction of a gold $\left(\mathrm{HAuCl}_{4}\right)$ precursor with ice cold
$\mathrm{NaBH}_{4}$ in the presence of trisodium citrate. Then, the seed solution was injected into the growth solution containing CTAB, $\mathrm{HAuCl}_{4}, \mathrm{AgNO}_{3}, \mathrm{HCl}$, and AA (Zhu et al., 2017). Synthesis of $\mathrm{Au} \mathrm{NBP/mesoporous} \mathrm{silica}\left(\mathrm{mSiO}_{2}\right)$ involved coating of the silica [through tetraethyl orthosilicate (TEOS)] in sideline of Au NBPs surface with the help of effective blocking of methoxy poly(ethylene glycol) (mPEG-SH) to the ends. mPEG-SH has a tendency to bind preferentially to the ends of Au NBPs, which is very important in further side growth of silica on the surface of $\mathrm{Au}$ NBPs. The synthesized anisotropic $\mathrm{Au} \mathrm{NBP} / \mathrm{mSiO}_{2}$ further used as a hard template for position selective deposition of Pd on $\mathrm{Au}$ NBPs. The schematic representation for the synthesis of $\mathrm{Au} \mathrm{NBP} /$ side-Pd nanostructures is shown in Figures 3A,B shows TEM image of the $\mathrm{Au} \mathrm{NBP}, \mathrm{Au} \mathrm{NBP} /$ end- $\mathrm{mSiO}_{2}, \mathrm{Au}$ $\mathrm{NBP} /$ end- $\mathrm{dSiO}_{2}$, and $\mathrm{Au} \mathrm{NBP} /$ side-Pd nanostructure. In addition to the small amount of TEOS on $\mathrm{mSiO}_{2}$ components, there was sequential deposition of dense $\mathrm{SiO}_{2}$ at the end. Finally, succeeding Pd deposition was directed by the dense silica components that occurred on the side surface of the Au NBPs.

\section{Janus Star-Sphere Nanoparticles}

Janus nanostructures that possess two or more different surface areas containing different optical and electronic properties are interesting candidates in diverse technological and biomedical applications. Janus nanoparticles with an iron oxide nanosphere and a branched gold nanostar (referred as Janus magnetic nanostar) was successfully synthesized by the two step seed-mediated-growth method (Reguera et al., 2017). Gold nanospheres were synthesized first and used as seeds for the growth of gold-iron oxide nanodumbbells. Subsequently, it acted as seeds for the directional growth of asymmetric gold nanostars: (1) The synthesis of asymmetric nano dumbbells involves a reaction between 1-octadecane, oleylamine, 1,2-hexadecanediol, oleic acid, and the two metal precursor $\mathrm{Fe}(\mathrm{CO})_{5}, \mathrm{HAuCl}_{4}$ at $300^{\circ} \mathrm{C}$ under $\mathrm{N}_{2}$ atmosphere. (2) The Janus magnetic nanostars synthesized by injecting nano dumbbells solution redispersed in chloroform with carboxyl terminated polyethylene glycol (PEG) into $\mathrm{N}, \mathrm{N}$-dimethylformamide (DMF) gold solution containing PVP after $1 \mathrm{~h}$ of stirring. The addition of carboxyl-terminatedPEG to the nanodumbbells provides a good dispersion in DMF that evades the obscurity in phase-transformation of nanodumbbells, as these are stabilized by oleic acid and oleylamine in water.

\section{Galvanic Synthesis Method}

Among the diverse methods available to achieve highly ordered anisotropic nanostructures, galvanic replacement reactions (GRR) has emerged a powerful method for molecular engineering which provides diverse opportunity for fabrication of anisotropic BNS with porous walls and hollow interiors. The term galvanic was derived from an Italian physician, Luigi Galvani, a biologist, philosopher, and pioneer of bioelectricity who established the first galvanic cell in the year 1780 (Focaccia and Simili, 2007). The GRR consists of a redox process between two metals where the lesser noble metal has the tendency to reduce the more noble metal cation having a higher redox potential with a suitable driving force (Liu and Astruc, 2017). The driving force here developed from the reduction potential 


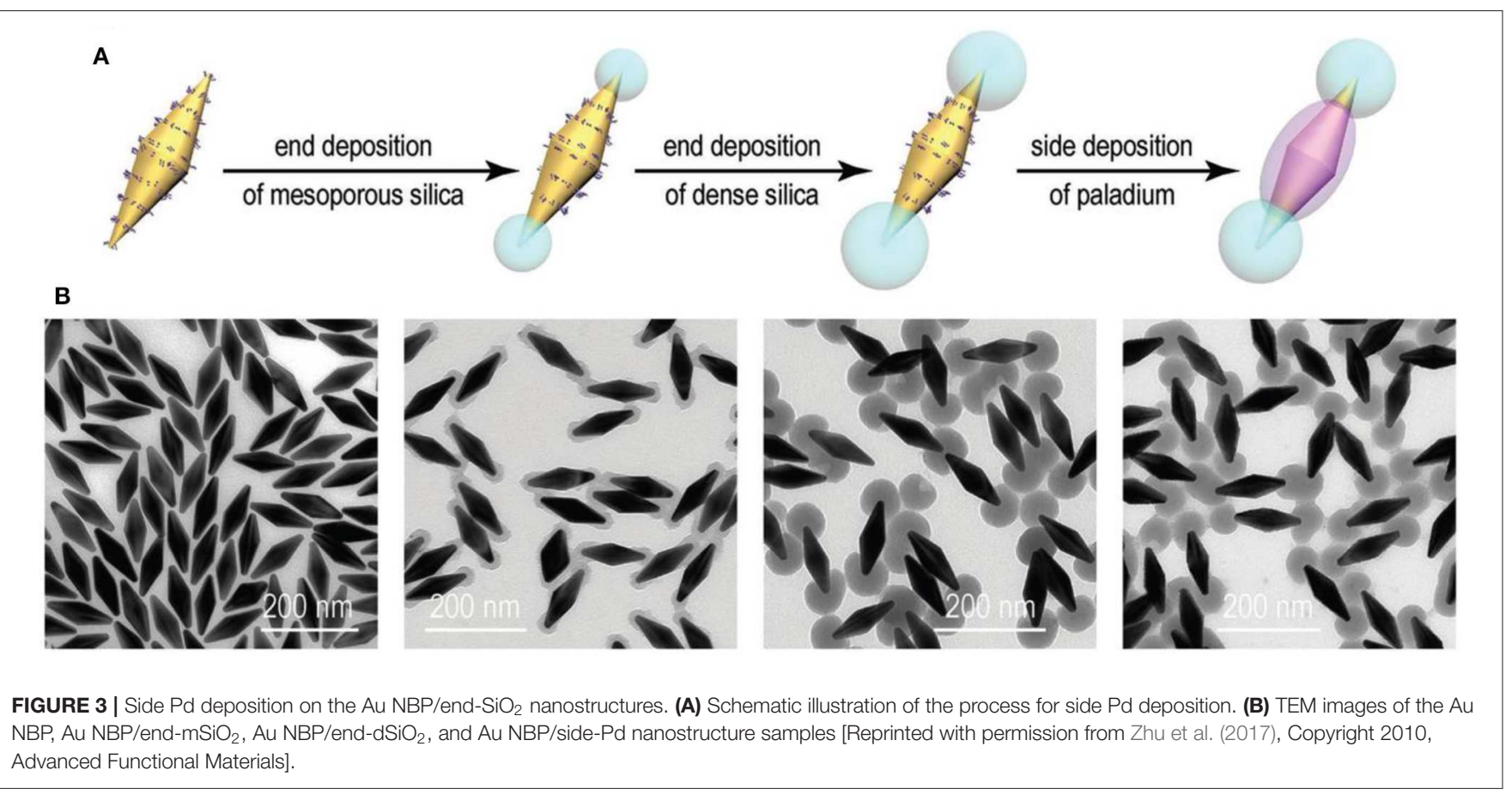

difference between the two different metals where one acts as a sacrificial template and the other is metal cation (Da Silva et al., 2017). The sacrificed metal is corroded when it interacts with the ions of the second metal in the solution phase. This effortless reaction path can be used for the selection of metal ions to synthesize diverse anisotropic BNS. Manipulating the precursor feed ratio, the final product composition and morphology can be tuned. The shape of the nanostructures can be customized by providing a sacrificial template of distinct geometries (Da Silva et al., 2017). The template allows for restrictive oxidation and dissolution of atoms from particular sites because these particular sites have different chemical reactivities. This difference arises due to the shielding of the template by the deposition of the second metal during GRR, forming a layer at a specific location. Consequently, deposition of the metal atom on the template forms a porous shell with the same morphology, resembling its parent template with a slight rise in the dimensions.

When a metal cation interacts with a metal that has a lower reduction potential with respect to itself, it prefers to reduce first via the standard GRR route and accumulate in the core (Sun and Xia, 2002, 2004). For example, GRR between Ag NPs and $\mathrm{AuCl}_{4}^{-}$ ions, two half equations occur as follows (Lu et al., 2007; Da Silva et al., 2017).

$$
\begin{array}{r}
\mathrm{Ag}_{(\mathrm{aq})}^{+}+1 \mathrm{e}^{-} \rightarrow \mathrm{Ag}^{0} \varepsilon_{1}^{0}=0.8 \mathrm{~V} \\
\mathrm{AuCl}_{4^{-}(\mathrm{aq})}+3 \mathrm{e}^{-} \rightarrow \mathrm{Au}^{0}+4 \mathrm{Cl}_{(\mathrm{aq})}^{-} \varepsilon_{1}^{0}=1.0 \mathrm{~V}
\end{array}
$$

Resulting the final balanced equation:

$$
\mathrm{AuCl}_{4^{-}(\mathrm{aq})}+3 \mathrm{Ag}^{0} \rightarrow 3 \mathrm{Ag}_{(\mathrm{aq})}^{+}+\mathrm{Au}^{0}+4 \mathrm{Cl}_{(\mathrm{aq})}^{-} \varepsilon_{1}^{0}=0.2 \mathrm{~V}
$$

Ag NPs in contact with $\mathrm{AuCl}_{4}^{-}$in solution, the $\mathrm{Ag}^{0}$ oxidized to $\mathrm{Ag}_{(\mathrm{aq})}^{+}$ions and $\mathrm{AuCl}_{4}^{-}$reduced to $\mathrm{Au}^{0}$ as shown in reaction (3), due to GRR driven by the electrochemical potential difference facilitating in the formation of cavities. Reduced $\mathrm{Au}^{0}$ atoms accumulated on the surface of Ag NP. As both Ag and Au have a face centered cubic (fcc) crystal structure and very close lattices constants ( $\mathrm{a}_{\text {gold }}=4.079 \AA$; $\mathrm{a}_{\text {silver }}=4.086 \AA$ ), they easily form an Ag-Au alloy (Sun and Xia, 2004). A wide variety of nanostructures of $\mathrm{Au}, \mathrm{Pd}$, and $\mathrm{Pt}$ with unlike properties have been synthesized using different geometries of $\mathrm{Ag}$ as the template (Prevo et al., 2008; Zhang W. et al., 2012; Xia et al., 2013). A novel cellular like gold nanofeet was synthesized by a galvanic replacement reaction between $\mathrm{Au} @ \mathrm{Ag}$ core-shell and $\mathrm{Au}^{+}$. The $\mathrm{Ag}$ from the surface reduces the $\mathrm{Au}^{+}$resulting in further growth of the Au shells on the Au/Ag core shell nanorods (Zheng G. et al., 2012). GRR, combining with co-reduction and Kirkendall processes, are used to synthesize wide varieties of nanostructures of different surface morphologies (González et al., 2011; Liu et al., 2013; Nafria et al., 2016). A few examples discussed below provide a better understanding to the classic GRR mechanism.

\section{Nanosphere}

Monodispersed Co-Cu NPs similar to a spherical shape with controlled metal ratios using a facile GRR technique were studied (Nafria et al., 2016). At first, Co NPs were synthesized using trioctylphosphine oxide (TOPO), oleylamine (OLA), and 1,2 anhydrous dichlorobenze (DCB) and were degassed in an airfree vacuum environment. Further, the solution was heated up to $180^{\circ} \mathrm{C}$ with rapid injection of $\mathrm{Co}_{2}(\mathrm{CO})_{8}$ dissolved in DCB. The synthesis of the bimetallic involved injection of different stock solutions of $\mathrm{Cu}^{+}$ions in OLA to Co spherical NPs and was heated up to $180^{\circ} \mathrm{C}$. Upon the addition of $\mathrm{Cu}^{+}$to Co NPs, 
Co oxidized to $\mathrm{Co}^{2+}$ and a $\mathrm{Cu}$ shell was grown due to GRR. The driving force for the substitution of $\mathrm{Co}$ by $\mathrm{Cu}^{+}$ions was due to the superior reduction potential of $\mathrm{Cu}^{+}$than $\mathrm{Co}^{2+}$ (at $25{ }^{\circ} \mathrm{C}$ and $\left.1 \mathrm{~atm}, E^{\circ}\left(\mathrm{Co}^{2+} / \mathrm{Co}\right)=-0.28 \mathrm{~V} ; E^{\circ}\left(\mathrm{Cu}^{+} / \mathrm{Cu}\right)=0.52 \mathrm{~V}\right)$. TEM images of the bimetallic Co-Cu NPs is shown in Figure 4. The average size of Co NPs was $10 \mathrm{~nm}$ and, with the addition of $\mathrm{Cu}^{+}$the size slightly increased from Co NPs as shown in Figure 4A. HRTEM image (Figure 4B) confirmed the presence of Moire fringes in Co-Cu NPs, concluding that two crystalline structures are confined together. The planes of (011) and (111) with d-spacing of 2.44 and $2.98 \AA$ matches with $\mathrm{Cu}_{2} \mathrm{O}$ shell. The elemental mapping of bimetallic Co-Cu NPs in Figure 4C shows Co was confined in the core whereas partially oxidized $\mathrm{Cu}$ resided in the shell.

\section{Nanoboxes}

Porous hollow structures are very important due to two important features. One is the open hollow interior that allows access inside of the nanostructures and second is the distribution of metal atoms different from bulk nanostructures. The known fact is that porosity in the material develops due to differential solid-state diffusion rates of the reactants involved during the oxidation or alloying reaction. Due to this difference, it develops an interfacial motion between the two participating metals, which is known as Kirkendall effect. This effect was reported by Smigelkas and Kirkendall in the year 1947 for copper and zinc in brass, due to the difference in the diffusion rates of these two metals resulting in the movement of the interface (Paul, 2004). The atomic diffusion is accompanied by vacancy exchange and not by the interchange of atoms. When there is flow of matter in a particular direction, it is balanced by the reverse flow of vacancies which reduces into the formation of pores. Edgar González and co combined the mechanism of galvanic exchange and Kirkendall growth technique to form very unique structures of PdAuAg nanoboxes of a double-walled morphology (González et al., 2011). In this protocol, Ag was used as a template and $\mathrm{Au}, \mathrm{Pd}$, and Pt served as oxidizing agents in order to synthesize hollow nanostructures. The synthesis of trimetallic nanoboxes involved three steps: (a) Mixture of CTAB and AA added into aqueous solution containing silver metal precursor; (b) Addition of second metal precursor, $\mathrm{HAuCl}_{4}$ with a flow rate from 20 to $50 \mu \mathrm{L} / \mathrm{min}$; and (c) Addition of Pd salt with a flow rate from $200 \mu \mathrm{L} / \mathrm{min}$ to $270 \mu \mathrm{L} / \mathrm{min}$ and followed by isolation of synthesized nanostructures.

Initially oxidation of Ag was favored since it has less positive reduction potential. $\mathrm{Ag}$ was oxidized and $\mathrm{Au}^{3+}$ was reduced since the electron transferred from the less noble metal Ag and was accepted by $\mathrm{Au}^{3+}$, as both $\mathrm{Au}$ and $\mathrm{Ag}$ have a fcc crystal structure and very close lattice constant value. $\mathrm{Au}^{0}$ deposits over $\mathrm{Ag}$, forming a fine thin layer. Formation of the thin layer over $\mathrm{Ag}$ restricts further oxidation of Ag. As the area of the electrode increases, the corrosion becomes stronger in the part of Ag which is exposed and uncovered by $\mathrm{Au}^{0}$, resulting in the formation of pinholes forming void in Ag cubes. Thus, a galvanic replacement takes place between $\mathrm{Ag}$ and $\mathrm{Au}$. The released electron migrates to the surface of the nanocube and is attracted by $\mathrm{AuCl}_{2}^{-}$, resulting in $\mathrm{Au}^{0}$ growth further on the $\mathrm{Ag}$ template due to preferential nucleation. After the formation of cavities via GRR and the successive formation of Au coating, growth of a thin film by Ag starts due to diffusion intermetallic coupling resulting in the Kirkendall cavity. Trimetallic structures of $\mathrm{Pd}-\mathrm{Au}-\mathrm{Ag}$ synthesized with the same mechanism by the addition of both $\mathrm{Au}$ and Pd precursors simultaneously or sequentially as shown in Figure 5.

\section{Nanotubes}

The synthesis of PtAg nanotubes occurred via the spontaneous galvanic displacement (SGD) method, where Ag nanowires (NWs) were displaced by Pt (Strand et al., 2016). The report explains the mechanism for the formation of Ag nanowires using $\mathrm{K}_{2} \mathrm{PtCl}_{4}$. The growth of an uneven surface over the nanotubes was facilitated by the replacement of $\mathrm{Ag}$ by $\mathrm{Pt}$ during SGD reaction. In the synthesis, commercially available Ag NWs were used as a displacement template during the SGD reaction by Pt. Initially, Ag NWs were dispersed in the DI water (1:1 w/V ratio) and heated to $90^{\circ} \mathrm{C}$ in the oil bath. Then the solution was injected with an aqueous solution of $\mathrm{K}_{2} \mathrm{PtCl}_{4}$ and the reaction was carried out in different intervals of time $(0,2,5,10,15,60$, and 120 minutes) in order to determine the displacement mechanisms. Figure 6 shows the morphology of the nanotubes obtained from the SGD reaction and the elemental mapping revealed the greater part of displacement of $\mathrm{Ag}$ by Pt had occurred between 2 to $10 \mathrm{~min}$ with an increase in Pt wt\% from time $=0$ to $10 \mathrm{~min}$.

\section{Antigalvanic Reduction Method}

As discussed, GRR phenomenon is a spontaneous process, when atoms of one metal come into contact with another metal ion of a higher electrochemical potential in comparison to the metal atom in solution. The metal atoms oxidize and go into the solution phase and then the simultaneous reduction of metal ions are reduced to atoms and deposit over the metal template. Thus, diversity of template choice in the GRR method can result in an extensive range of nanostructures ( $\mathrm{Wu}, 2012)$. Recently, a new phenomenon, antigalvanic reduction reaction (AGR), which contradicts to the galvanic principle, has been more appealing for the synthesis of BNS. Advanced research revealed that AGR is more relevant to mechanistic, synthetic, and catalytic purposes. The AGR mechanism deals with the metals ions which are lesser noble (more reactive) reduced by more noble metals (less reactive). This is completely opposite to the GRR and has not been explored widely. Murray and co-workers in the year 2010 revealed that an $\mathrm{Au}$ nanocluster $\left[\mathrm{Au}_{25}\left(\mathrm{SC}_{2} \mathrm{H}_{4} \mathrm{Ph}\right)_{18}\right]$ reacts with $\mathrm{Ag}$ ions (Choi et al., 2010), follows the AGR mechanism. The wellknown nanocluster $\mathrm{Au}_{25} \mathrm{~L}_{18}$ i.e., $\left[\mathrm{Au}_{25}\left(\mathrm{SC}_{2} \mathrm{H}_{4} \mathrm{Ph}\right)_{18}\right]$, Ligand $\mathrm{L}=\mathrm{SC}_{2} \mathrm{Ph}$ (phenylethanethiolate) holds 13-atom icosahedral core surrounded by $6 \mathrm{Au}_{2}(\mathrm{SR})_{3}$ shell. When it reacts with $\mathrm{Ag}^{+}$, it forms $\left.\left.\mathrm{Au}_{25} \mathrm{Ag}\left(\mathrm{SC}_{2} \mathrm{H}_{4}\right) \mathrm{Ph}\right)_{18}, \mathrm{Au}_{24} \mathrm{Ag}_{1}\left(\mathrm{SC}_{2} \mathrm{H}_{4}\right) \mathrm{Ph}\right)_{18}$, and $\left.\mathrm{Au}_{23} \mathrm{Ag}_{2}\left(\mathrm{SC}_{2} \mathrm{H}_{4}\right) \mathrm{Ph}\right)_{18}$ with respect to an optimized ratio of $\mathrm{Ag}^{+} / \mathrm{Au}_{25} \mathrm{~L}_{18}^{-}$. Au atoms reduced by $\mathrm{Ag}^{+}$without any alteration of ligands confirms the AGR phenomena.

The first general theory of the AGR mechanism was predicted in 2012 by $\mathrm{Wu}$ et al., and stated that the thiolate ligands in $\left[\mathrm{Au}_{25}\left(\mathrm{SC}_{2} \mathrm{H}_{4} \mathrm{Ph}\right)_{18}\right]$ might be responsible for supporting the 


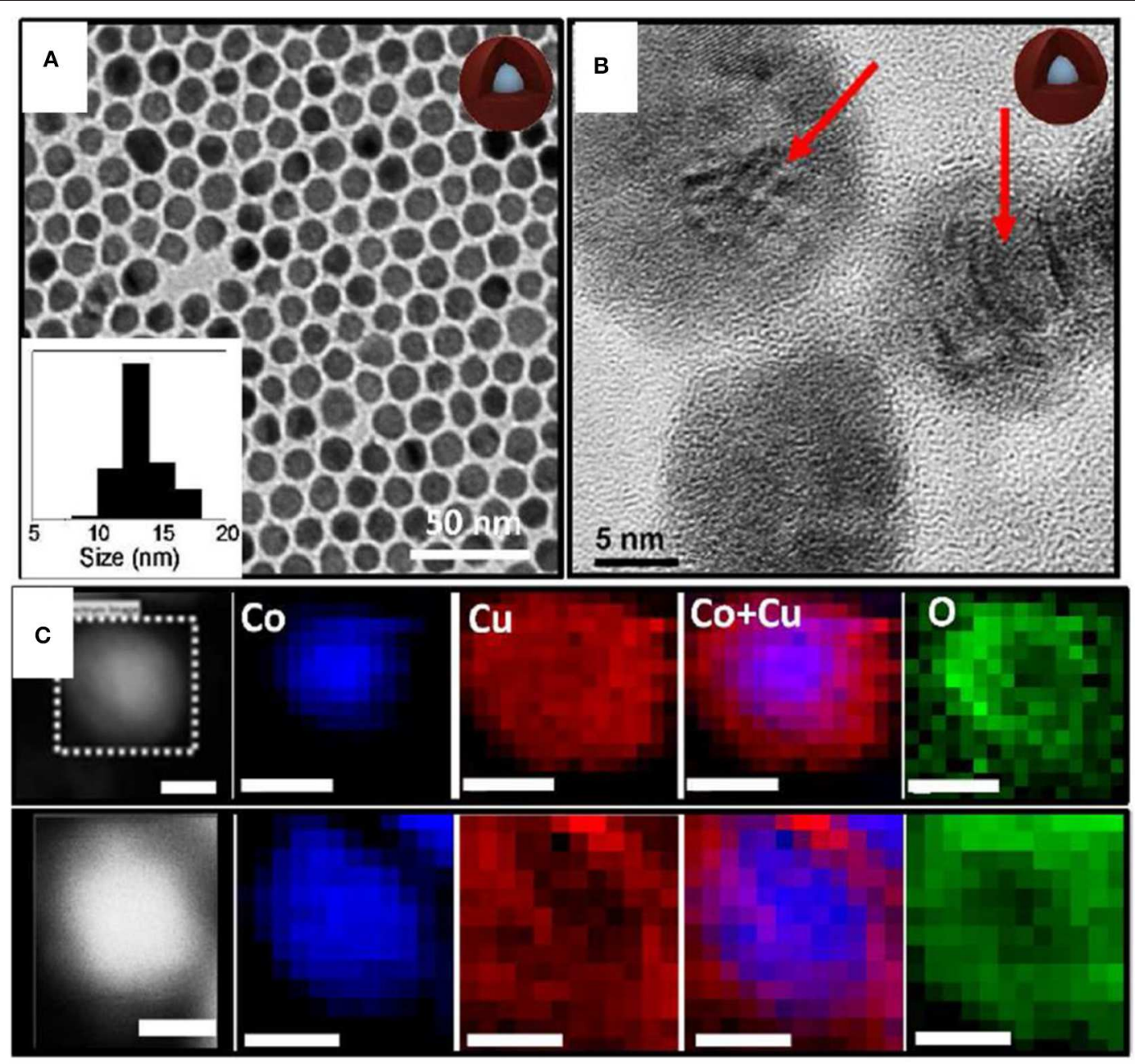

FIGURE 4 | (A) TEM micrographs and size histograms (insets) of the $\mathrm{CO}_{0.6}$-Cu 0.4 NPs. (B) HRTEM micrograph of Co-Cu NPs. (C) Two examples of HAADF (Z contrast) images and $\mathrm{Co}, \mathrm{Cu}$, and $\mathrm{O}$ electron energy loss spectroscopy (EELS) compositional maps of $\mathrm{CO}_{0.6}-\mathrm{Cu}_{0.4} \mathrm{NPs}$. Scale bars correspond to 5 nm [Reprinted with permission from Nafria et al. (2016), Copyright 2016, Langmuir].

AGR mechanism (Wu, 2012). The ligands attached on the surface of nanocrystals develop a partial negative charge to the Au and Ag nanocrystals. Wang and Wu suggested that in the AGR mechanism there is no need for reductive ligands as nanoparticles can reduce metal ions (Wang et al., 2014). Small NPs of more noble metals, such as $\mathrm{Au}$ and $\mathrm{Pt}$, can reduce less noble metal ions like $\mathrm{Ag}^{+}$, but this is a deviation from the conventional free energy-based arguments used in galvanic displacement $(\mathrm{Wu}$, 2012; Wang et al., 2014). Sun and coworkers studied a one pot approach using the AGR mechanism for an Ag-Au nanocluster (Sun et al., 2014), whereas Corpuz reported positively charged photoluminescent bimetallic Au-Ag nanoclusters synthesized based on the AGR mechanism (Corpuz et al., 2017). A core shell nanostructure of silver (shell) and gold (core), Ag@Au NPs synthesized and explained through AGR mechanism (Sahu and Prasad, 2015). This was carried out in order to determine whether the reduction phenomena of $\mathrm{Ag}^{+}$is driven by ligand dodecylamine $(\mathrm{DDA})$ or the surface atoms $\left(\mathrm{Au}^{0}\right)$ of $\mathrm{Au} \mathrm{NPs}$ acting as reducing agents in AGR reaction. The reaction was carried out using two different capping agents, dodecanethiol (DDT) and DDA. When DDT was used as a capping agent on $\mathrm{Au}$ NPs, there was no core shell formation. Instead individual $\mathrm{Au}$ and Ag nanoparticles resulted. But when DDA was capped on Au NPs, an Ag@Au core shell nanostructure formed. Hence, it is concluded that the reduction of $\mathrm{Ag}^{+}$to $\mathrm{Ag}^{0}$ on the surface of $\mathrm{Au}^{0}$ was governed by DDA resulting in the final core-shell structure. The change in the ligand results in alterations in the nanostructures. Even if DDA has a stronger binding affinity with $\mathrm{Au}$ NP surface, during reflux condition DDA detaches from $\mathrm{Au}$ NPs leaving behind a naked Au NP exposed surface. Meanwhile, DDA does not possess binding affinity with an $\mathrm{Ag}^{0}$ surface which results in the generation of nascent uncapped $\mathrm{Ag}^{0}$ atoms in the solution. The nascent uncapped $\mathrm{Ag}^{0}$ atoms preferred to attach to the surface of naked Au NP resulting in an Ag@Au core shell nanostructure. On the other hand, when DDT was used as a capping agent, it possessed a strong affinity toward both $\mathrm{Ag}$ and 


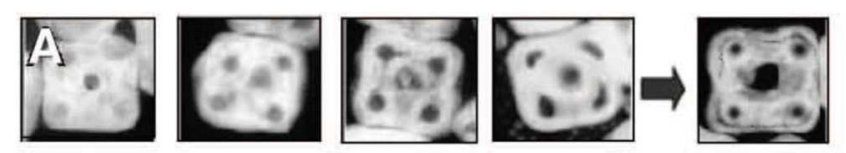

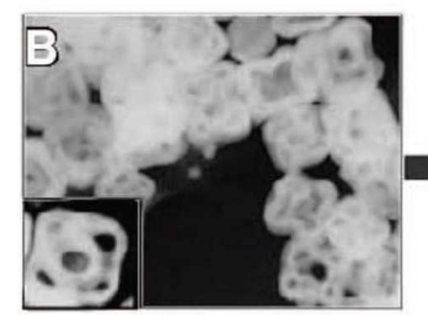

Pd-Ag
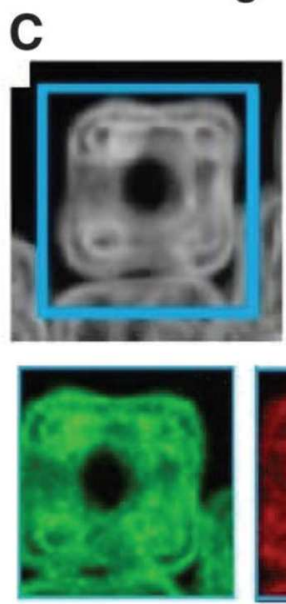
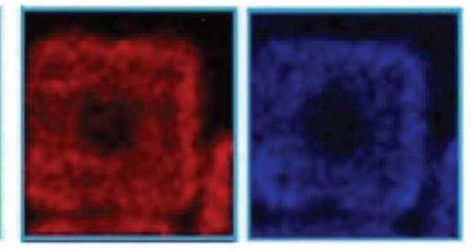

$\mathrm{Au}$

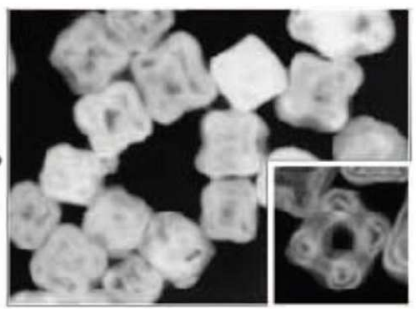

Au-Pd-Ag

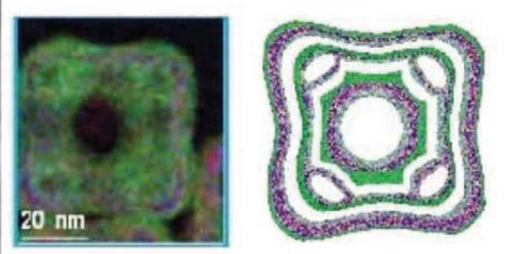

$\mathrm{Ag}$

Pd
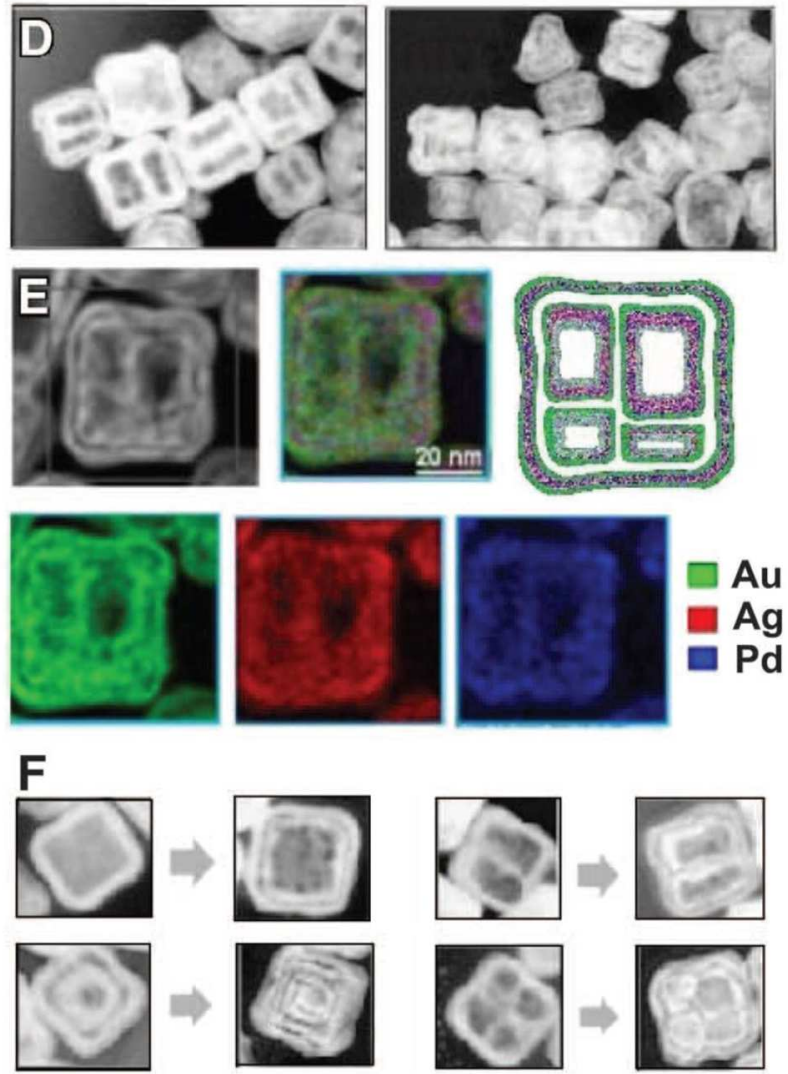

FIGURE 5 | (A) TEM images illustrating the stages of formation of hollow nanostructures of Au-Ag-Pd nanoboxes by sequential action of galvanic replacement and Kirkendall effect. (B) TEM images showing the nanostructures dominated by galvanic replacement using Pd (left) and by Kirkendall effect using Au (right). (C) HAADF-STEM image and corresponding EDX elemental maps. (D) TEM images of multichambered NPs. (E) HAADF-STEM detail and corresponding EDX elemental maps. (F) TEM images of different structures produced by sequential action of galvanic replacement and Kirkendall effect [Reprinted with permission from González et al. (2011), Copyright 2011, Science].

Au nanoparticle surfaces. After the reduction of $\mathrm{Ag}^{+}$ions to $\mathrm{Ag}^{0}$, DDT immediately caps $\mathrm{Ag}^{0}$ atoms in the solution, resulting in individual $\mathrm{Au}$ and $\mathrm{Ag}$ nanoparticles. The nature of ligand binding to the nanoparticle has a significant role in establishing the final structure.

\section{One Pot Synthesis Method}

The synthesis of BNS involved two mechanisms: GRR and the co-reduction of two metal precursors in the presence of a reducing agent. However, GRR requires significant redox potential difference between two metals to drive the reaction which limits the feasible combinations of two metals and tunability in its elemental composition. Whereas, the second approach involves the tiresome cleaning process after synthesis. Thus, in order to improve the efficiency of any chemical reaction researchers tried one pot techniques in which reactants are subjected to successive chemical reactions in one reactor involving multiple steps. These methods have been mostly preferred due to the elimination of the prolonged separation procedure and purification of the additional compounds which can be done in less time and increases the yield of the reaction. Further, this method eases the reaction path in the preparation of BNS. However, it needs a harsh reaction environment for controlling the nucleation of metals that have different reducing behaviors. Using a one-step synthesis route, core-shell structures can be synthesized when the two metals have large differences in their standard redox potentials by providing a harsh reaction environment (He et al., 2010; Park et al., 2014).

\section{Trigonal Bipyramid Nanoframes}

Nanoframes have an ultrathin structure of large surface-tovolume ratios and high surface areas (Chen et al., 2015) and come in various sizes where the active atoms are more exposed over the surface. Hierarchical trigonal bipyramid nanoframes (HTBNFs) are formed using a $\mathrm{Pt}-\mathrm{Cu}$ formed alloy using one pot synthesis method (Chen et al., 2015). The structure consists of a string of parallel structural units with sharp branch tips. $\mathrm{CuCl}_{2}, \mathrm{H}_{2} \mathrm{PtCl}_{6}$, and $\mathrm{KI}$ were added altogether with polyvinylpyrrolidone (PVP) and ethylene glycol and heated at $140^{\circ} \mathrm{C}$ for the formation of Pt-Cu HTBNFs. In this synthesis, the co-reduction of the 


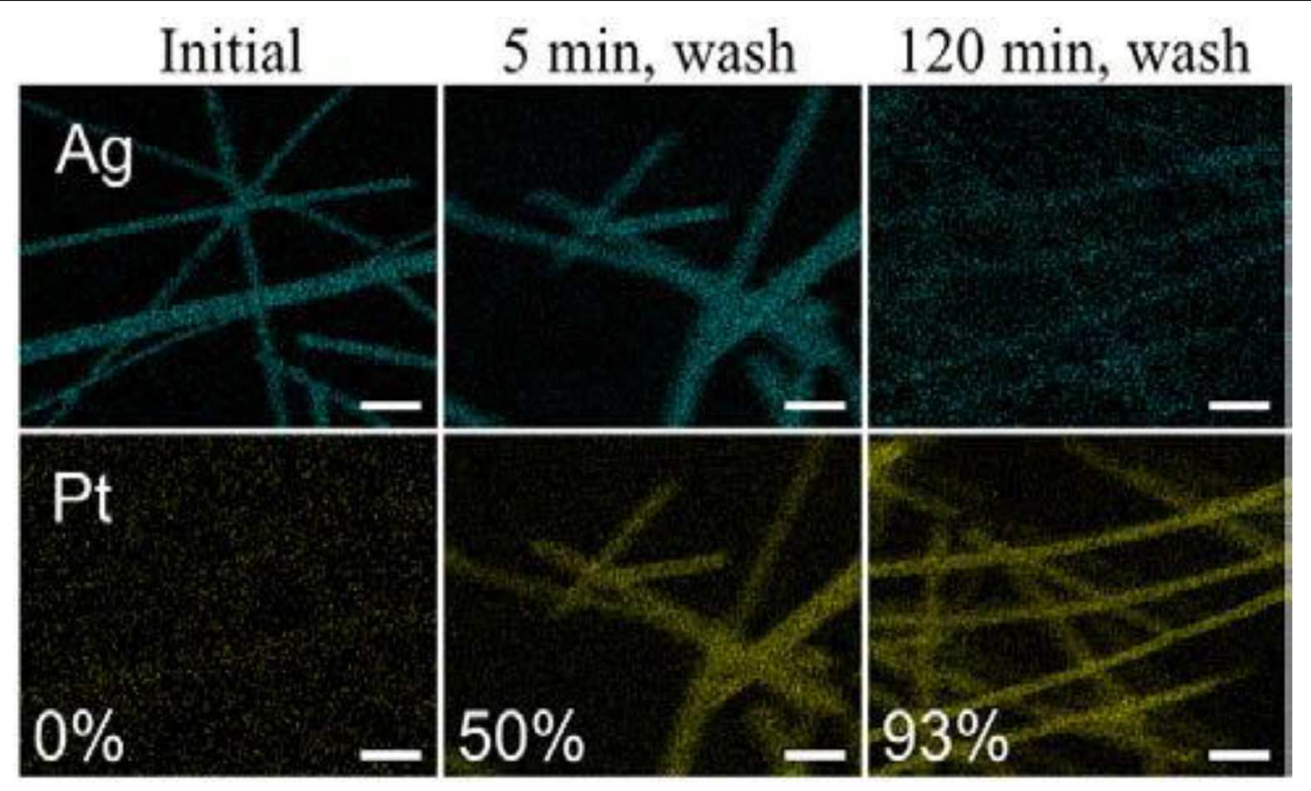

FIGURE 6 | Elemental mapping from SEM of PtAg nanotubes made via SGD of Ag nanowires after reaction times of 0, 5, and 120 min, with Pt wt\% listed. The 5- and 120-min samples were washed with a sodium chloride $(\mathrm{NaCl})$ solution then refluxed in $\mathrm{HNO}_{3}$ to remove residual Ag on the surfaces of the nanotubes. Scalebars are $500 \mathrm{~nm}$ [Reprinted with permission from Strand et al. (2016), Copyright 2016, The Journal of Physical Chemistry].

precursor metals $\mathrm{Pt}$ and $\mathrm{Cu}$ lead the shape of alloy structure. Due to the large differences of the electrochemical reduction potential of $\mathrm{PtCl}_{6}^{2-} / \mathrm{Pt}^{0}$ and $\mathrm{Cu}^{2+/} \mathrm{Cu}^{0}, \mathrm{I}^{-}$forms a complex by coordinating with $\mathrm{Pt}^{4+}$ rather than $\mathrm{Cl}^{-}$, resulting in a closer reduction potential between $\left[\mathrm{PtI}_{6}\right]^{2-} / \mathrm{Pt}^{0}$ and $\mathrm{Cu}^{2+} / \mathrm{Cu}^{0}$. The size of the HTBNFs was influenced by the amount of KI added in the synthesis. With the gradual addition of the KI solution, the size of the HTBNFs decreased from 250 to $110 \mathrm{~nm}$ as shown in Figure 7. Initially, miniature monopods, bipods, and tripods shaped structures were formed, and the development of secondary, tertiary, and higher-organized branches were formed confirming the final HTBNF structures. Metal precursors $\left(\mathrm{H}_{2} \mathrm{PtCl}_{6}\right.$ and $\left.\mathrm{CuCl}_{2}\right)$ ratio played a vital role in the development of different Pt-Cu HTBNFs.

\section{Nano Dendritic Core-Shell}

Various protocols have been used for the synthesis of Pt-based bimetallic core-shell nanospheres using $\mathrm{Pt}$ as a shell. Coreshell nanostructures where Pt develops as a dendritic shell have been studied. A Pt based bimetallic core-shell required an elevated temperature and pressure to optimize the synthesis protocol. Catalytic activity of the Pt-based core shell depends on the thickness of the shell. The thinner the Pt shell, the better the catalytic activity. Using the one pot inexpensive synthesis method, Au-Pt nanocolloids with $\mathrm{Au}$ as core and $\mathrm{Pt}$ as a dendritic shell was synthesized (Ataee-Esfahani et al., 2010). Au-Pt nanocolloids formed by mixing chloroplatinic acid hexahydrate $\left(\mathrm{H}_{2} \mathrm{PtCl}_{6} \cdot 6 \mathrm{H}_{2} \mathrm{O}\right)$ and $\mathrm{HAuCl}_{4}$ precursors and growth was controlled by the low concentration of surfactant with an ultrasonic irradiation. Altering the Pt and $\mathrm{Au}$ molar ratios in the reaction, the shell thickness has been controlled.
AA and pluronic F127 were used as a reducing agent and surfactant/structure directing agent, respectively. The formation of the core-shell is driven by the reduction potentials difference of the two dissimilar metal precursors, $\mathrm{H}_{2} \mathrm{PtCl}_{6}$ and $\mathrm{HAuCl}_{4}$. The reduction of $\mathrm{AuCl}_{4}^{-}$and $\left[\mathrm{PtCl}_{6}\right]^{2-}$ can be explained by the subsequent reactions as follows (Ataee-Esfahani et al., 2010).

$$
\begin{array}{r}
\mathrm{AuCl}_{4}^{-}+3 \mathrm{e}^{-} \rightarrow \mathrm{Au}+4 \mathrm{Cl}^{-}+1: 00 \mathrm{eV} \text { vs. SHE } \\
{\left[\mathrm{PtCl}_{6}\right]^{2-}+2 \mathrm{e}^{-} \rightarrow\left[\mathrm{PtCl}_{4}\right]^{2-}+2 \mathrm{Cl}^{-}+0.68 \mathrm{eV} \text { vs. SHE }} \\
{\left[\mathrm{PtCl}_{4}\right]^{2-}+2 \mathrm{e}^{-} \rightarrow \mathrm{Pt}+4 \mathrm{Cl}^{-}+0.76 \mathrm{eV} \text { vs. SHE }}
\end{array}
$$

Due to the large reduction potential difference between Au and $\mathrm{Pt}, \mathrm{Au}$ cations reduced faster to form Au core, while Pt cations reduced later and formed overgrowth as a dendrite structure on the surface of the Au core. Pluronic F127 acts as a structuraldirecting agent, resulting in crown-like ethers conformation (Wang et al., 2010).

The interaction linking hydrophobic polypropylene oxide (PPO) groups and the Pt surface is through adsorption on the $\mathrm{Pt}$ surface. Due to which cavities are formed following the formation of $\mathrm{Pt}$ dendrites, such phenomena is restricted in the case of $\mathrm{Au}$ as F127 cannot act as a capping agent due to $\mathrm{Au}$ core forming an irregular nanostructure. Figures $\mathbf{8 A , B}$ shows the TEM image of $\mathrm{Au}-\mathrm{Pt}$ nanocolloids. The size of $\mathrm{Au}-\mathrm{Pt}$ nanocolloids are in the range of 20-35 nm. HRTEM images in Figure 8C shows the lattice fringes of an individual Pt shell corresponds to (111) plane with face-centered cubic (fcc) crystal. A ring like pattern was observed in SAED pattern (Figure 8D) indicating the different planes such as (111), (200), (220), and (311) of Pt confirms 

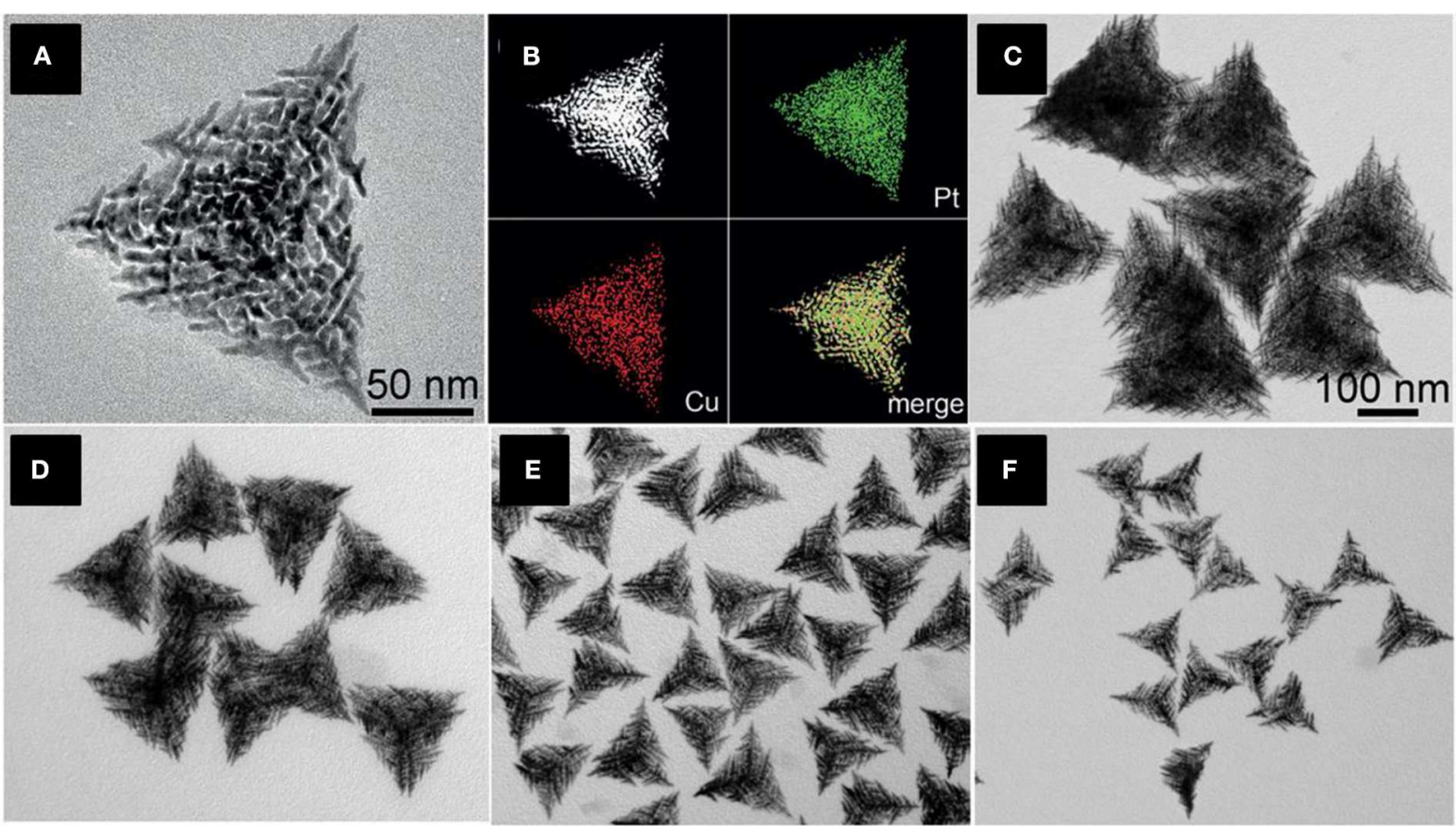

FIGURE 7 | (A) TEM image of an individual Pt-Cu HTBNFs, (B) The corresponding HAADF-STEM image and elemental mapping showing the distribution of Pt (green) and $\mathrm{Cu}(\mathrm{red})$. (C-F) TEM images of Pt-Cu HTBNFs obtained through the addition of different amounts of $\mathrm{Kl}$ aqueous solution in the synthesis while keeping the other parameters constant: (C) $30 \mathrm{~mL}-250 \mathrm{~nm}$, (D) $50 \mathrm{~mL}-200 \mathrm{~nm}$, (E) $100 \mathrm{~mL}-150 \mathrm{~nm}$, and (F) $150 \mathrm{~mL}-110 \mathrm{~nm}$. The scale bar in (C) also applies to (D-F) [Reprinted with permission from Chen et al. (2015), Copyright 2015, Chemie International Edition].
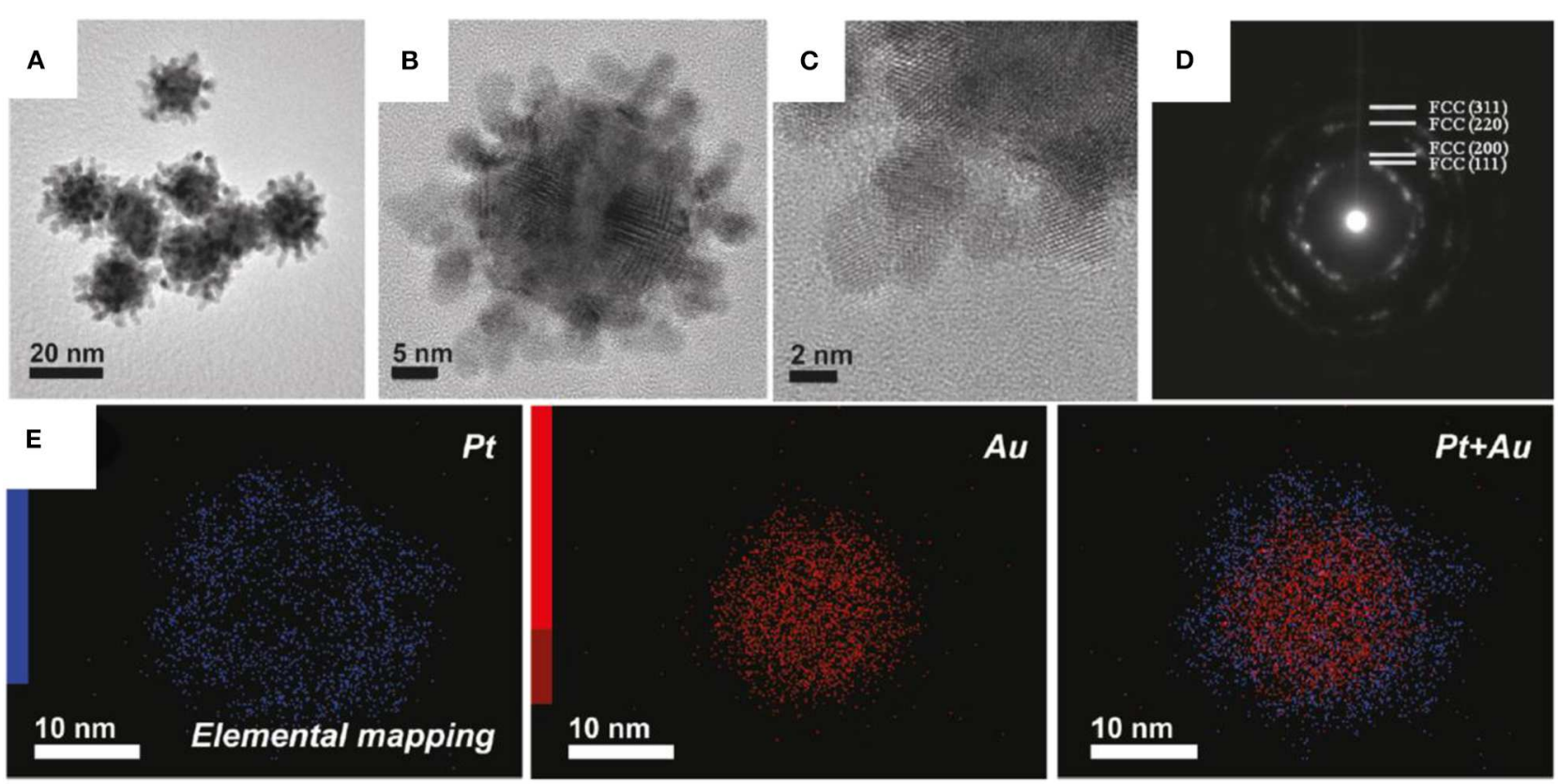

FIGURE 8 | (A) Low-magnification and (B) high-magnification TEM images of Au-Pt nanocolloids, (C) High-magnification TEM image of the Pt shell, (D) Selected-area electron diffraction (SAED) patterns taken from the Pt shell region, (E) elemental mapping of Au-Pt nanocolloids, the red color indicates Au, whereas the blue color indicates Pt [Reprinted with permission from Ataee-Esfahani et al. (2010), Copyright 2010, Chemistry of Materials]. 
the polycrystalline nature. Elemental mapping in the Figure $\mathbf{8 E}$ shows $\mathrm{Au}$ is present at the center as a core and $\mathrm{Pt}$ forms dendritic shell leading to an Au-Pt core-shell.

\section{Nano Dendritic Alloy}

Pt-Pd bimetallic nanodendrites were synthesized using the onepot solvothermal method as shown in Figure 9 (Wu et al., 2019a). Three different nanoporous Pt-Pd bimetallic nanodendrite structures were obtained by varying the precursor ratio of $\mathrm{Pt}$ and $\mathrm{Pd}$ in an ethylene glycol solution in the presence of iodide ions and polyvinylpyrrolidone (PVP). When the ratio between $\mathrm{Pt} / \mathrm{Pd}$ was maintained to $3: 1$ in the standard reaction, uniform porous spherical nanodendrites with particle sizes of $44.9 \pm$ $2.6 \mathrm{~nm}$ were formed. Further, changing the $\mathrm{Pt} / \mathrm{Pd}$ ratio to $2: 1$ and $1: 1$, the shape and size varied to nanodendrites with coarser branches of particle size of $34.3 \pm 2.1 \mathrm{~nm}$ and nanodendrites with quasi-cubic shape branches of particle size of $25.1 \pm 1.8 \mathrm{~nm}$, respectively. Here, Iodide ion has shown its vital role in the formation of dendrite structure. Absence of iodide ion results in NPs with uniform polyhedral nanostructures of particle size 4.2 $\pm 1.8 \mathrm{~nm}$. But in addition to $\mathrm{I}^{-}$to the reaction, $\mathrm{Pt}^{4+}$ and $\mathrm{Pd}^{2+}$ ions form complexes, $\left[\mathrm{PtI}_{6}\right]^{2-}$ and $\left[\mathrm{PdI}_{4}\right]^{2-}$. Iodide ions further alter the reduction sequence and decrease the reduction rate of metal precursors and formed Pt-Pd bimetallic nanodendrites. Dendritic alloy was reported by Kamel Eid and coworker in 2016 using the one pot co-reduction technique. The synthesized porous dendritic bimetallic Pt-Ni nanocrystals are highly efficient catalysts in oxygen reduction reaction (ORR) (Eid et al., 2016). The aqueous solution of $\mathrm{K}_{2} \mathrm{PtCl}_{4}, \mathrm{Ni}\left(\mathrm{NO}_{3}\right)_{2}$, PVP, and AA under ultrasonic irradiation or magnetic stirring created dendritic or flower like nanocrystals of $\mathrm{Pt}-\mathrm{Ni}$ synthesized, respectively. The synthesized structures were reported to show a very good electrocatalytic activity in ORR.

\section{Core-Shell Nanocubes}

Bimetallic nanocubes with high-surface energy facets were difficult to synthesize unless the two metal ions were coreduced in precisely under-controlled kinetic environments. The nucleation of metal and controlling of the kinetic growth in the presence of multiple metal precursors is the great challenge. Park and coworkers employed the one pot protocol in an aqueous room temperature solution and synthesized Au-Pd coreshell nanocubes (NCs) with a truncated hexoctahedral $(\mathrm{THOH})$ structure (Park et al., 2014). For the synthesis of THOH Au@Pd $\mathrm{NCs}$, a mixture of $\mathrm{KAuBr}_{4} \cdot \mathrm{xH}_{2} \mathrm{O}, \mathrm{K}_{2} \mathrm{PdBr}_{4}$, and CTAB in an aqueous solution was treated with $\mathrm{AA}$ and $\mathrm{NaOH}$ (molar ratio $4: 1$ ) to reduce two precursor metal ions. The solution was shaken for a few seconds and kept undisturbed for $1 \mathrm{~h}$. The synthesized solution was centrifuged to remove excess unreacted ions. The manipulation of the molar ratio among the two metals, reducing agents, and capping agent provided a control over the nucleation and the kinetic growth resulting in the formation of $\mathrm{THOH}$ Au@Pd NCs. The morphology restricted to the core shell because the Au precursor possesses higher reduction kinetics compared to the Pd precursor in the provided experimental condition. The Au precursor preferentially reduces first to form an Au NC core confined to hexoctahedral $(\mathrm{HOH})$ which are employed as seeds for further growth. Pd has an essential role in establishing high-index facets during the growth. In the absence of the $\mathrm{Pd}$ precursor, irregular morphology of $\mathrm{Au}$ NCs formed. This happens because Pd selectively grows on the sites of high energy facets of $\mathrm{Au}$ NCs resulting in an increase in the stabilization of high-index facets. As a result of this, the crystal structure kinetically entraps in the $\mathrm{HOH}$ structure (Lee et al., 2013).

\section{Nanoboxes}

The one pot facile synthesis technique was employed to synthesize AgAu nanoboxes (He et al., 2010). The synthesis proceeds with a mixture of two metal precursors, $\mathrm{HAuCl}_{4}$ and $\mathrm{AgNO}_{3}$ (molar ratio $1: 1$ ), in an aqueous solution and $\mathrm{AA}$ is immediately added under vigorous shaking. The appearance of a blue color confirms the formation of AgAu NPs stabilized by CTAB $(0.1 \mathrm{M})$. Ag/Au molar ratio is the key parameter to control the shape of AgAu NPs. Star-like solid NPs formed at a lower $\mathrm{Ag} / \mathrm{Au}$ molar ratio of 0.2 and increased the molar ratio of $\mathrm{Ag} / \mathrm{Au}$ from 0.4 to 0.8 , resulting in nanoboxes forming. Uniform nanoboxes are formed at an $\mathrm{Ag} / \mathrm{Au}$ ratio of $1 / 1$ (Figure 10). It confirms that the involvement of Ag is highly important in the formation AgAu nanoboxes.

Figures 10A,B shows the SEM and TEM images of AgAu NPs confined to a cubic morphology with holes over the surface in which the edge of the nanoboxes is more highly dense than the center. The regular size of nanoboxes is $75 \pm 5 \mathrm{~nm}$ with a shell thickness of $9.6 \pm 2.1 \mathrm{~nm}$. The electron diffraction (ED) pattern confirms (Figure 10C) the polycrystalline nature of the nanoboxes. The $\mathrm{d}$ spacing of lattice fringes near walls (Figure 10D) is 0.234 which corresponds to (111) plane of $\mathrm{Au}$ or (111) plane of $\mathrm{Ag}(0.233 \mathrm{~nm})$.

\section{One Pot Hydrothermal Technique}

The hydrothermal technique is the most important tool for the synthesis of anisotropic nanostructures (Byrappa and Adschiri, 2007). The hydrothermal technique not only helps in processing monodispersed and highly homogeneous nanostructures but also provides a reaction condition in order to acquire hybrid and nano-amalgamated materials. The technique involves a heterogeneous reaction and is subjected to high pressure and temperature in aqueous solvents which dissolve and recover the materials. In hydrothermal synthesis, all the reactants are dissolved in water or any other suitable solvent and sealed in a vessel for the synthesis of the desired shape, size, and structure (Byrappa and Adschiri, 2007). Products of low impurity, monodispersity, smaller particle size, high activity, and fewer defects with simple purification shows the advantages of the hydrothermal method over other synthetic approaches. Synthesis of Pt-Pd concave nanocubes (Wu et al., 2019b), Au-Pd alloy and core-shell (Kuai et al., 2012), and Pd-Rh nanoframes and cubes (Ye et al., 2015) have been synthesized through the hydrothermal method as discussed below.

\section{Concave Nanocubes}

$\mathrm{Pt}$ and Pd can be effortlessly shaped into a bimetallic alloy structure and both are known for their catalytic behavior. The catalytic performance to a large extent depends on the shape, 

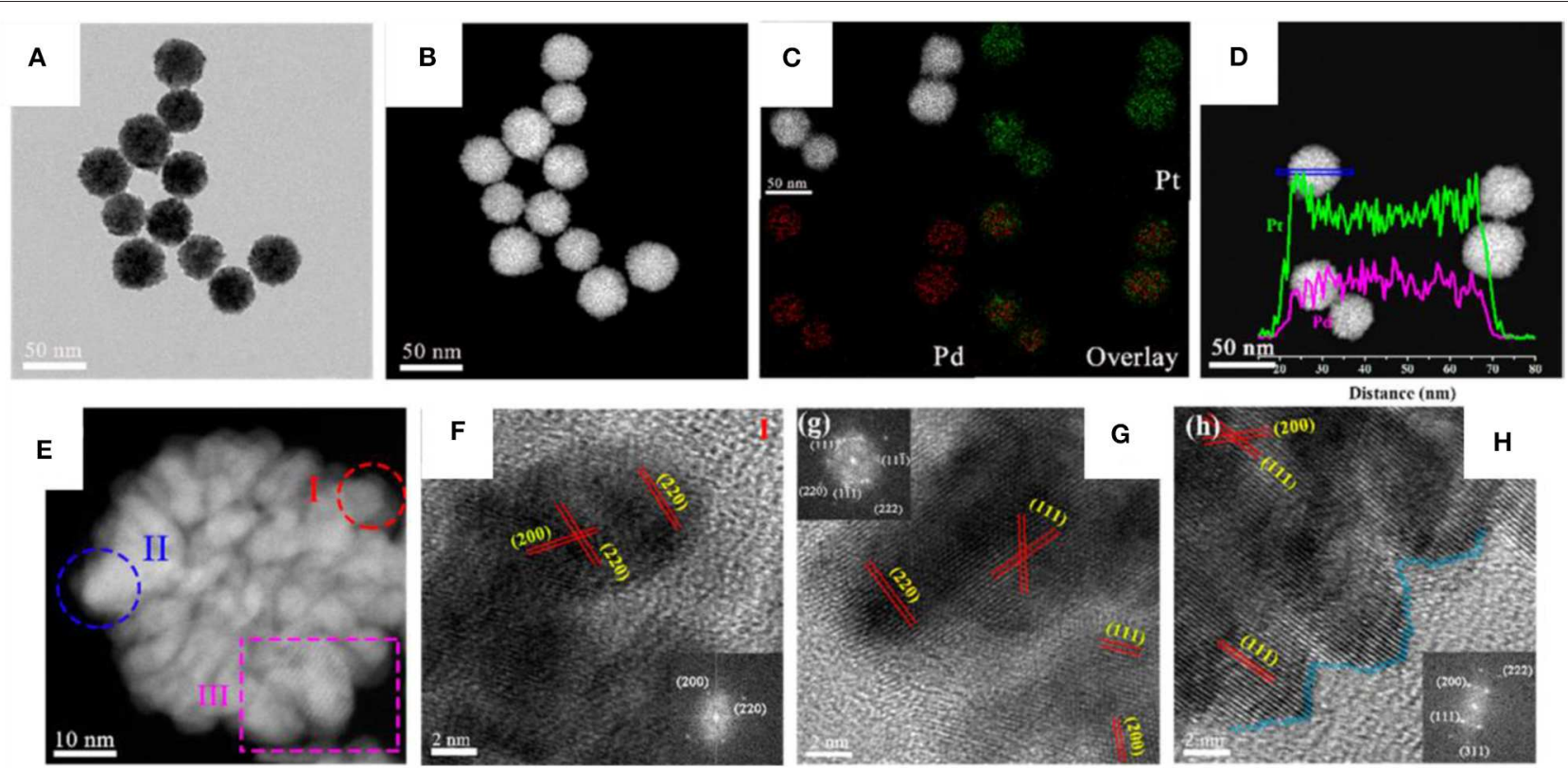

FIGURE 9 | (A) Bright-field TEM image and (B) HAADF-STEM image of $\mathrm{Pt}_{3} \mathrm{Pd}_{1}$ nanodendrites, (C) HAADF-STEM image and EDX elements mapping and (D) line-scanning profiles. (E) The individual $\mathrm{Pt}_{3} \mathrm{Pd}_{1}$ nanodendrites and $\mathbf{( F - H )}$ the corresponding local HR-TEM image. The insets in (F-H) show the corresponding FFT pattern [Reprinted with permission from Wu et al. (2019a), Copyright 2019, ACS Sustainable Chemistry and Engineering].
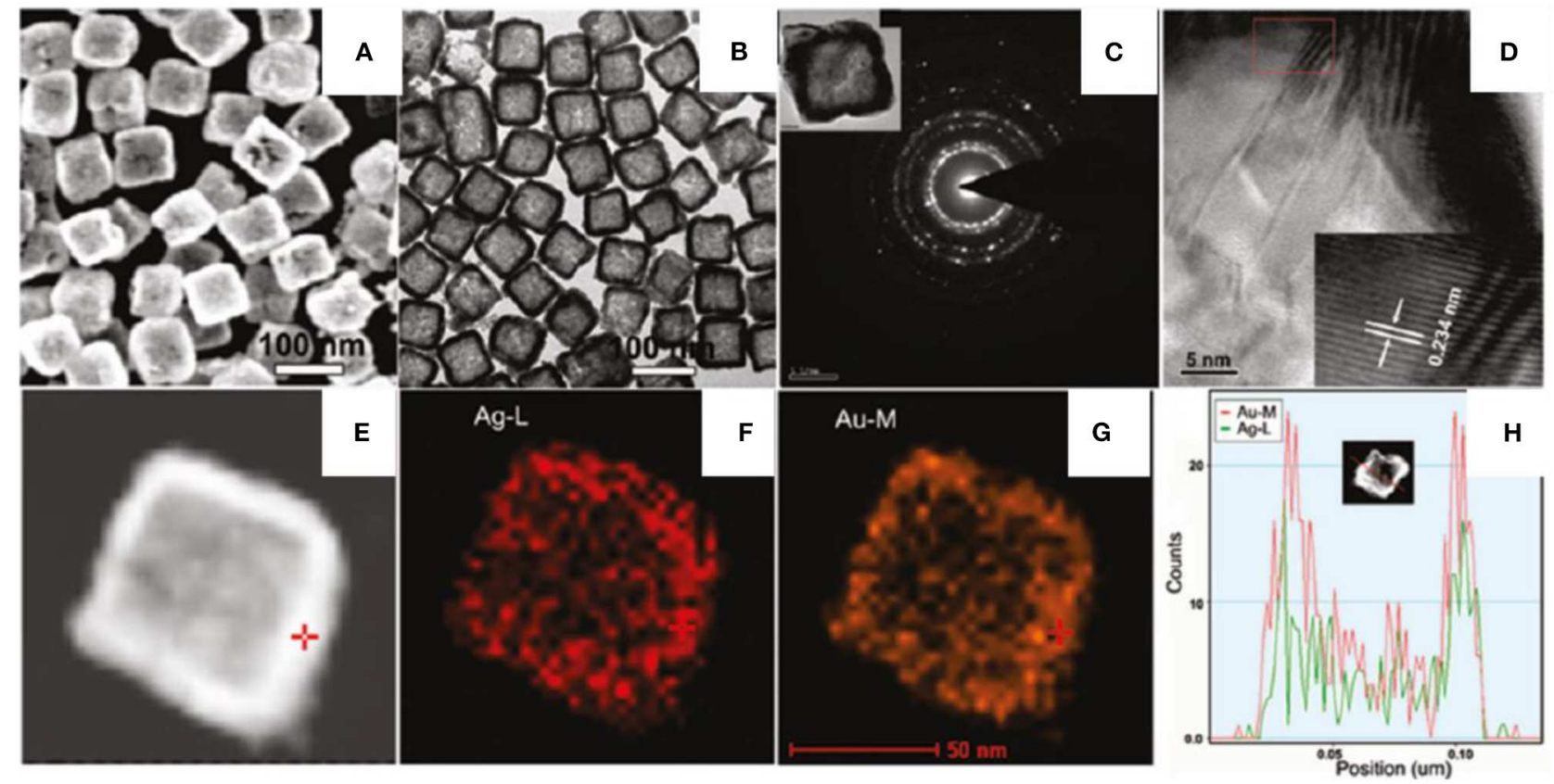

FIGURE 10 | (A) SEM and (B) TEM images of AgAu nanoboxes; (C) ED pattern of a single nanobox; (D) HRTEM image near the box wall; (E) STEM-HAADF image; (F,G) STEM-EDX element mappings of Ag and Au, respectively; and (H) cross-section composition line profiles of one nanobox [Reprinted with permission from He et al. (2010), Chemistry of Materials].

size, structure, and morphology of the bimetallic structure. A facile one pot method is used for Pt-Pd symmetry broken concave nanocubes (SBCNCs) synthesis (Wu et al., 2019b). A solution of disodium tetrachloropalladate $\left(\mathrm{Na}_{2} \mathrm{PdCl}_{4}\right), \mathrm{H}_{2} \mathrm{PtCl}_{6} \cdot 6 \mathrm{H}_{2} \mathrm{O}$, and sodium iodide (NaI) in $\mathrm{N}$-dimethylformamide (DMF) was used for the hydrothermal reaction at $130^{\circ} \mathrm{C}$. Pd (II) cannot 


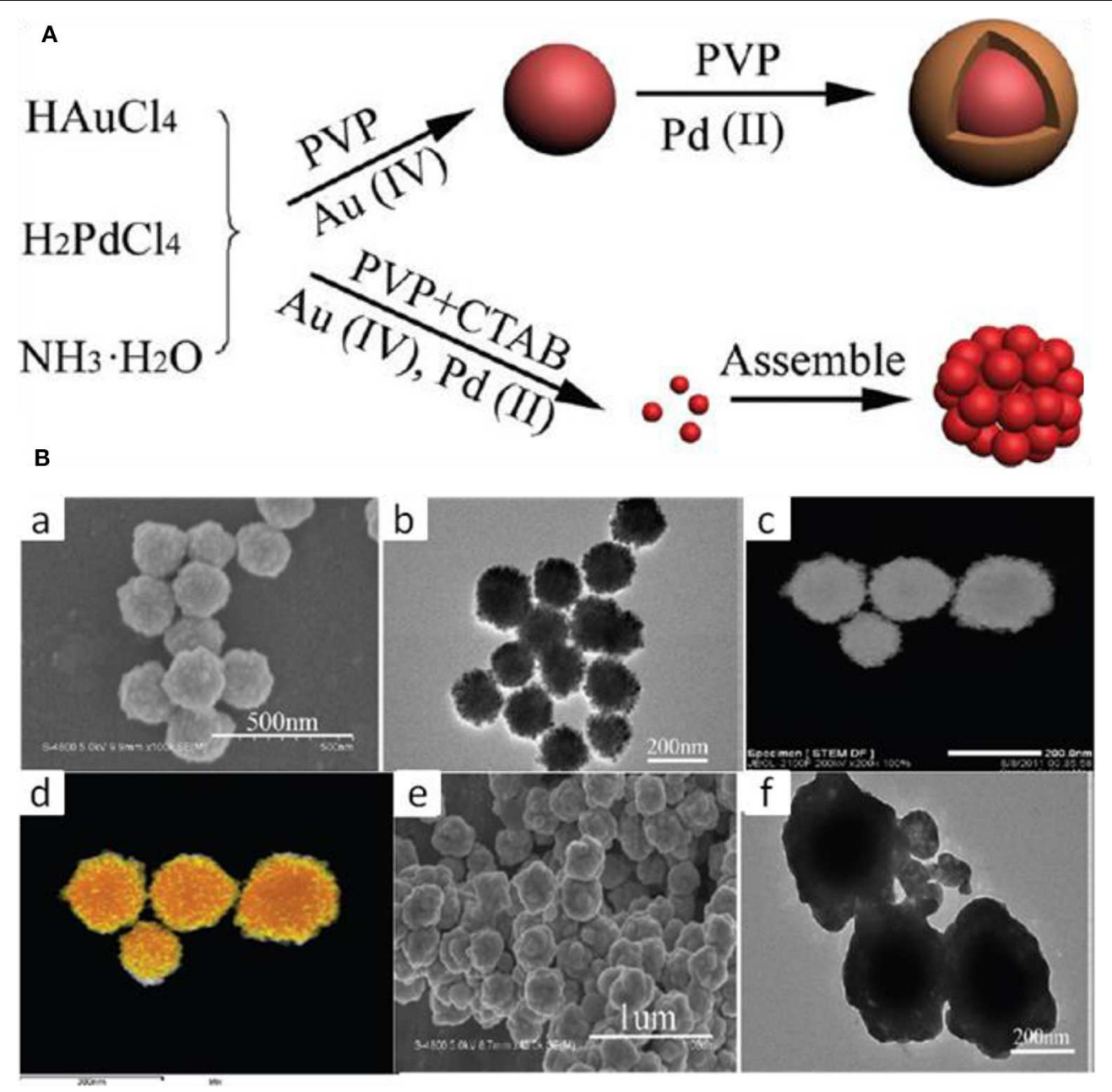

FIGURE 11 | (A) The formation Process of Au-Pd Core-Shell and Alloy BNS, (B) Morphology analysis, (a) SEM, (b) TEM, (c) HAADF-STEM, and (d) HAADFSTEM-EDS mapping images of Au-Pd alloy nanostructures. (e) SEM and (f) TEM images of Au-Pd core-shell nanostructures [Reprinted with permission from Kuai et al. (2012), Copyright 2012, Langmuir].

be reduced before $\mathrm{Pt}$ (IV) due to their respective standard reduction potentials $\left(\mathrm{E}^{0}\right)$ between $\mathrm{Pd}^{2+} / \mathrm{Pd}$ and $\mathrm{Pt}^{4+} / \mathrm{Pt}$. As iodide ion and $\mathrm{Pt}$ (IV) forms a complex in an aqueous medium, favored by aqua and hydroxo substitution (Huang et al., 2009; Straney et al., 2014; Zhang J. et al., 2016), and hence, it is easier to reduce $\mathrm{Pd}$ before $\mathrm{Pt}$. Capping agent $\mathrm{I}^{-}$prefers to grow along (100) direction forming Pt-Pd nanocubes in the beginning. But an unstirred solution provides a slow surface diffusion rate which escorts the directional growth toward the vertexes and stacks atoms to the corner of the nanocubes, leading to the arrangement of asymmetric concave nanocubes.

\section{Alloy and Core-Shell}

To Minimize the two-step epitaxial growth mechanism which has the limitation of large-scale synthesis, Long Kuai and co approached the one pot hydrothermal co-reduction technique for the synthesis of Au-Pd alloy and core-shell nanostructures (Kuai et al., 2012).
$\mathrm{HAuCl}_{4}$ and $\mathrm{H}_{2} \mathrm{PdCl}_{4}$ were used as the metal precursors and PVP as the reductant for the synthesis of core-shell nanostructures. The addition of CTAB to the reaction containing $\mathrm{HAuCl}_{4}, \mathrm{H}_{2} \mathrm{PdCl}_{4}$, and PVP alloy structure formed instead of core-shell. The size of the core-shell was larger than the alloy, as shown in Figure 11. Hence, the reaction proceeds core-shell formation naturally, but the addition of CTAB restricts the formation of core-shell and favors the formation of alloy. This is due to the stronger reducing activity of CTAB than PVP under hydrothermal conditions and both the metals reduced together, forming alloy nanostructures.

\section{Nanoframes and Nanoboxes}

Hollow metallic nanostructures are a significant research area due to their large surface areas with high reactivity (Sun and Xia, 2004). Both Pd and Rh act as significant catalysts and have been hardly studied in terms of bimetallic hollow nanostructures. Ye and coworkers reported the synthesis of Pd$\mathrm{Rh}$ nanoframes and cubes using a hydrothermal reaction (Ye 
et al., 2015). Pd-Rh bimetallic nanoframes and nanoboxes were produced by selectively removing Pd cores from different Pd$\mathrm{Rh}$ nanocubes. The average Pd-Rh nanocube size was $12.7 \pm$ $1.3 \mathrm{~nm}$. Further, selective elimination of Pd from the synthesized Pd-Rh nanocubes formed the cubic nanoframes. The size and shape of the nanoframes was intact from the parent nanocubes but the edges were fairly thin (i.e., $2 \mathrm{~nm}$ ). During the synthesis, formaldehyde ( $\mathrm{HCHO}$ ) was used for altering the growth behavior and reaction kinetics to form different hollow nanostructures, nanoframes, or nanoboxes. The shape of Pd-Rh nanocubes was intact in the absence of $\mathrm{HCHO}$. However, the size increased to $33.0 \pm 3.8 \mathrm{~nm}$ due to the reduced nucleation from the weak reducing agent $\mathrm{PVP}$. $\mathrm{Rh}-\mathrm{Rh}$ has a strong interaction during etching and has more stability than Pd (Lu et al., 2013). As a result, Pd comes out preferentially from the nanocubes. However, $\mathrm{Pd}$ cannot be entirely removed from the nanocubes as Pd partly alloys with $\mathrm{Rh}$ throughout the synthesis of $\mathrm{Pd}-\mathrm{Rh}$ nanocubes. After drawing Pd from Pd-Rh nanocubes, hollow structures were obtained (Xie et al., 2012).

\section{Nanorods}

$\mathrm{Ni}$-Fe bimetallic phosphides (NiFeP) nanorods were synthesized at a low-temperature phosphorization process using a hydrothermal technique (Du et al., 2018). In the method, a $\mathrm{Ni}-\mathrm{Fe}$ bimetallic-organic frameworks (MIL-88- $\mathrm{Fe}_{2} \mathrm{Ni} \mathrm{MOF}$ ) precursor was used to engineer NiFeP nanorod structures. The atoms of $\mathrm{Ni}$ and $\mathrm{Fe}$ were connected with the help of an organic ligand terephthalic acid. The procedure follows: (1) synthesis of the MIL-88- $\mathrm{Fe}_{2} \mathrm{Ni}$ nanorods precursor in which hydrothermal treatment of mixture $\mathrm{FeCl}_{3} .6 \mathrm{H}_{2} \mathrm{O}, \mathrm{Ni}\left(\mathrm{NO}_{3}\right)_{2} .6 \mathrm{H}_{2} \mathrm{O}, 1,4$ benzenedicarboxylic acid $\left(\mathrm{H}_{2} \mathrm{bdc}\right)$ in dimethyl formamide (DMF), and $\mathrm{NaOH}$ solution were carried out at $100{ }^{\circ} \mathrm{C}$ for $15 \mathrm{~h}$. (2) The synthesized MIL- $88-\mathrm{Fe}_{2} \mathrm{Ni}$ nanorods was further calcined at $450{ }^{\circ} \mathrm{C}$ forming $\mathrm{NiFe}_{2} \mathrm{O}_{4}$ nanorods. (3) The final $\mathrm{NiFeP}$ was obtained by low temperature phosphorization of $\mathrm{NiFe}_{2} \mathrm{O}_{4}$ nanorods with $\mathrm{NaH}_{2} \mathrm{PO}_{2}$ as phosphorus source. The NiFeP nanorods showed outstanding electrocatalytic behavior during the hydrogen evolution reaction (HER) in basic medium. A synergistic effect developed from $\mathrm{NiP}_{2}$ and $\mathrm{FeP}$ was responsible for the excellent electrocatalytic performance. The $\mathrm{Ni}$-Fe bimetallic phosphides $(\mathrm{NiFeP})$ nanorods form an alloy of well distributed $\mathrm{Fe}, \mathrm{Ni}$, and $\mathrm{P}$ elements.

\section{Electrospinning Technique}

The electrospinning technique is essentially used for fiber synthesis. Electric force is used to draw charged threads of material solutions or material melts up to fiber diameters in the order of a 100 nanometers using a polymer solution. This technique is widely recognized as a nano structuring technique because of its ability to direct the morphology and exterior topology to form fine fibers (Agarwal et al., 2009). There are two electrospinning arrangements: vertical and horizontal. Certain complex setups have been designed in order to get intricate nanofibrous structures with uniformity. The technique is maintained at room temperature under atmospheric conditions (Bhardwaj and Kundu, 2010). The system consists of three key apparatus: a high voltage power supplier, a spinneret, and a collecting plate which is grounded. It supplies a charge of a certain polarity to a polymer solution from a high voltage source. This is then collected by a collector of opposite polarity. The polymer fluid is supplied to the capillary tube for electrospinning and results in the formation of fiber. In order to achieve parallel bimetallic fibers, the whirling solution consists of two precursor salts with polymer compounds. Within polymer nanofibers, the reduction of salt produces NPs of diameters in the range of 3-5 nm. The dispersion of monometallic or bimetallic NPs in polymer nanofibers is of great interest for heterogeneous catalysis (Demir et al., 2004). In addition to the morphology, alteration of the elemental ratio in the mother solution provides wide tunability in the application using this technique.

\section{Nanofibers}

Carbon materials have attracted huge interest owing to their outstanding physicochemical properties studied as an electrocatalyst in fuel cells (Senthilkumar et al., 2018). Due to their high surface to volume ratio, carbon nanofibers have been widely used for extensive chemical characteristics (Ghouri et al., 2015).

Doping metal or bimetals with the carbon nanofibers enhances its properties. Pd-Cerium (Ce) bimetallic doped with carbon nanofibers synthesized using the electrospinning technique showed excellent electrochemical properties and high uniformity (Alvi and Akhtar, 2016). Cobalt (Co) has been recognized for its catalytic behavior from all other nonnoble metal catalysts and $\mathrm{Pd}$, being cheaper than $\mathrm{Pt}$ and also widely used as catalyst. Bimetallic nanofibers of $\mathrm{Pd}$-doped $\mathrm{Co}$ nanofibers offered excellent photocatalytic behavior and were used as the active material for diode (Barakat et al., 2012). Hui reported bimetallic $\mathrm{Ni}-\mathrm{Fe}$ oxide/carbon composite nanofibers with excellent OER catalytic behavior synthesized through the electrospinning technique. The advantage of this technique is the elemental proportions that can be precisely adjusted in the mother solution, which enhanced the OER activity (Chen et al., 2016). The molar ratio of $\mathrm{Fe}$ and $\mathrm{Ni}$ tuned to obtain a number of composite materials of different $\mathrm{Ni} / \mathrm{Fe}$ atomic ratios for various structures. The whole process is demonstrated in Figures 12I,IIA-B shows the SEM and TEM image of synthesized $\mathrm{Ni}-\mathrm{Fe}$ composite nanofibers calcined at $250^{\circ} \mathrm{C}$ and elemental mapping in Figures 12IIC-H shows the presence of $\mathrm{Ni}, \mathrm{Fe}, \mathrm{O}$, $\mathrm{C}$, and $\mathrm{N}$ in the composite material. The elements homogenously formed an alloy. The presence of nitrogen in large amounts obtained from PVP can easily be converted into N-doped carbon.

Magnetic anisotropic nanostructure has garnered great interest as it strongly influences the shape of hysteresis loop and, hence, controls the coercivity and remanence. Among magnetic nanostructures, $\mathrm{Co}-\mathrm{Ni}$ has gained more attention since it is capable of overcoming the super magnetic edge and further improve the contrast in magnetic resonance imaging. Co-Ni bimetallic fine nanofibers were synthesized by the electrospinning technique (Barakat et al., 2010). The synthesis followed mixing of two metal precursors, nickel acetate tetrahydrate and cobalt acetate tetrahydrate, in an aqueous solution in a 1:1 ratio and then dissolved in poly(vinyl alcohol $/ \mathrm{H}_{2} \mathrm{O}$ solution. The solution was allowed to stir for 
(I)
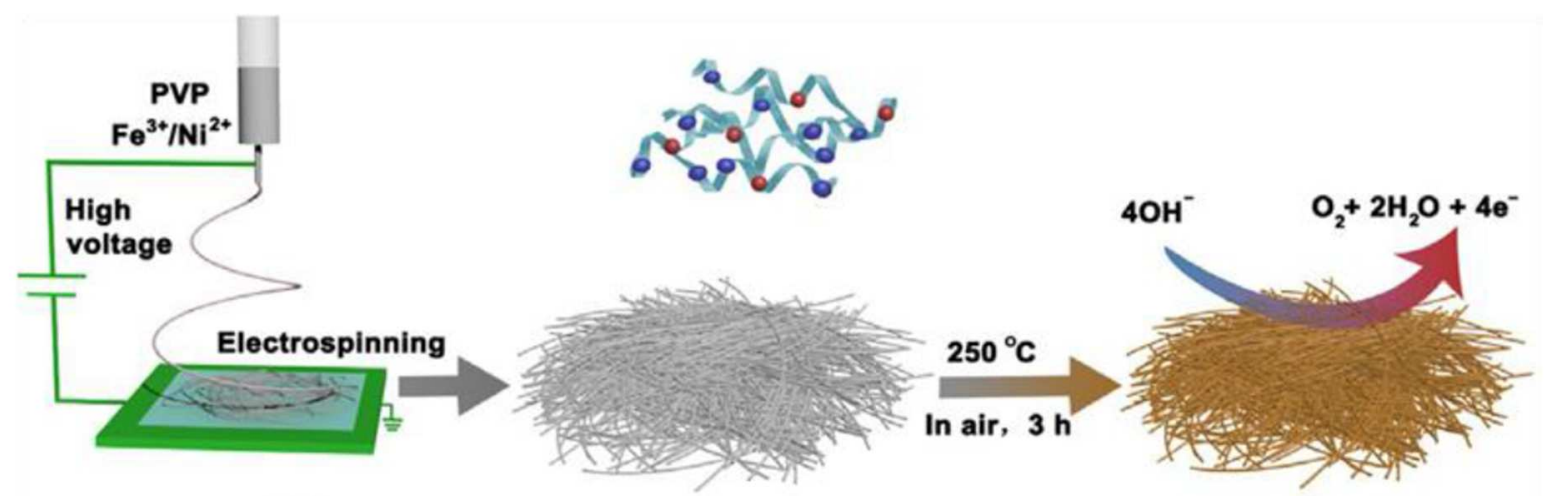

(II)
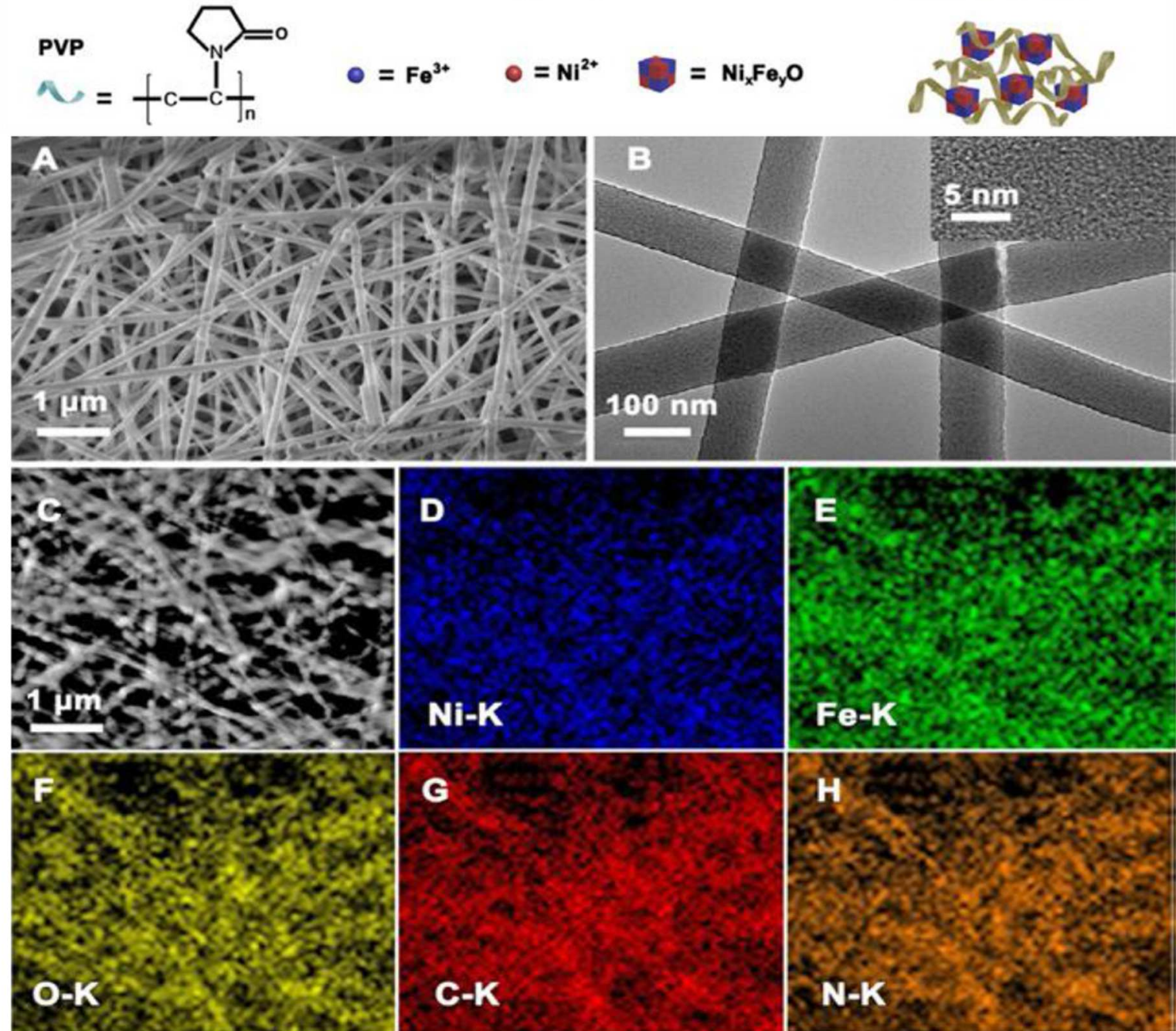

FIGURE 12 | (I) Illustration of the synthesis of the Ni-Fe oxide/carbon composite nanofibers. (II) (A) SEM image and (B) TEM image and a high-resolution TEM image (inset) of $\mathrm{Ni}_{1} \mathrm{Fe}_{2}-250$. (C) Scanning transmission electron micrograph and (D-H) the corresponding elemental mapping images of $\mathrm{Ni}_{1} \mathrm{Fe}_{2}-250[\mathrm{Reprinted} \mathrm{with}$ permission from Chen et al. (2016), Copyright 2016, ChemCatChem].

$5 \mathrm{~h}$ at $52^{\circ} \mathrm{C}$. A voltage of $20 \mathrm{kV}$ was applied to the solution through a high voltage generator. The solution is present in the capillary with an optimized inclination angle. A ground iron drum immersed in polyethylene sheet used as a counter electrode.
The synthesized nanofibers were further dried for $24 \mathrm{~h}$ and allowed to calcined at $800^{\circ} \mathrm{C}$ for $5 \mathrm{~h}$ in an argon atmosphere with a heating rate of $2.3^{\circ} \mathrm{C} / \mathrm{min}$. Figure 13 shows the FESEM image of synthesized Co-Ni nanofibers in different magnifications. 


\section{APPLICATION OF ANISOTROPIC BIMETALLIC NANOSTRUCTURE IN CATALYSIS}

A catalyst acts as the initiator to enhance the reaction rate by decreasing the threshold barrier, which helps to progress the reaction in less time and at a lower temperature and pressure. Basically, it decreases the activation energy to convert the reactant into the product faster. Furthermore, catalytic performance has been significantly developed with the advent of nanotechnology, which made it possible to use nanosized particles to catalyze various chemical reactions. Catalytic behavior absolutely depends on the shape, size, and morphology of the two metals. BNS have been extensively explored and are an interesting area of heterogeneous catalysis in many applications, especially for energy conversion and chemical transformations (Armbrster et al., 2010). Special interest in the synthesis of BNPs as catalyst is driven due to its alternation of electronic structure and generation of novel characteristics. When the second metal (guest metal) is added to the first metal (host metal) the combination of metals with its pristine property enhances catalytic activity, higher selectivity, and stability (Service, 2007). The combination of the guest metal with the host metal develops either: (a) an electronic effect or ligand effect, in which there is an alteration of electron properties or electronic arrangement in the active sites of the host metal due to an electron transfer from guest to host metals. The concept to estimate the influence of the guest metal over the host metal is the d-band center of

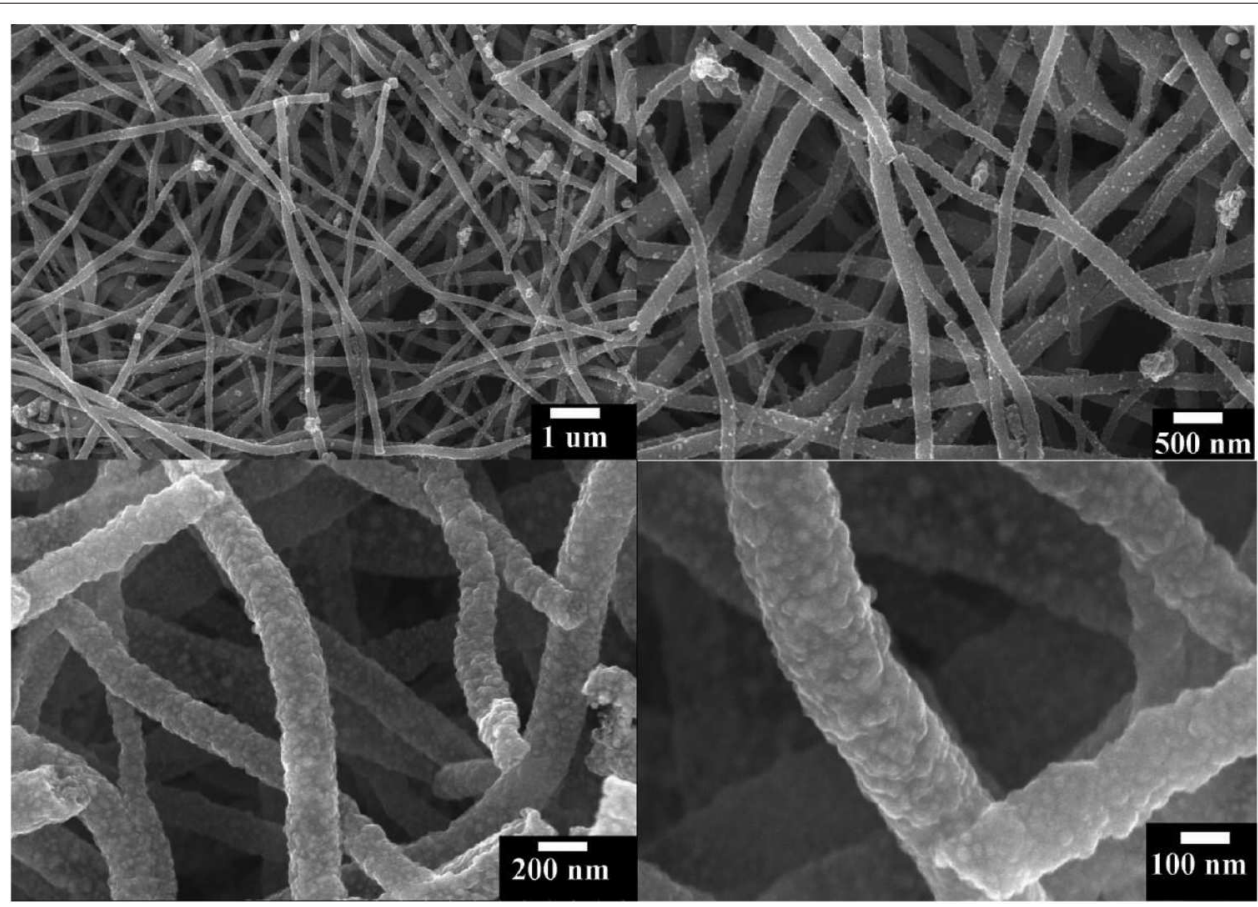

FIGURE 13 | Field emission scanning electron microscope (FESEM) images at different magnifications for the obtained powder after calcinations of the electrospun $\mathrm{NiAc/CoAc/PVA} \mathrm{nanofiber} \mathrm{mats} \mathrm{in} \mathrm{argon} \mathrm{atmosphere} \mathrm{at} 800^{\circ} \mathrm{C}$ for $5 \mathrm{~h}$ [Reprinted with permission from Barakat et al. (2010), Copyright 2010, The Journal of Physical Chemistry C].

TABLE 1 | Mentioned methods and BNS in different catalytic application.

\begin{tabular}{|c|c|c|c|}
\hline Material & Synthesis methods & Application in catalysis & References \\
\hline Nickel-Iron Phosphides Nanorods & Hydrothermal method & Hydrogen Evolution Reaction & Du et al., 2018 \\
\hline Pt-on-Pd Bimetallic Nanodendrites & Seed mediated growth strategy & Methanol oxidation reaction (MOR) & Kunz et al., 2017 \\
\hline Pd-Ir octapods and Nanocages & Galvanic replacement and co-reduction & Hydrazine decomposition & Liu et al., 2013 \\
\hline Pd crown-Au jewel Nanocluster & Galvanic replacement reaction & Aerobic glucose oxidation & Zhang H. et al., 2012 \\
\hline Au-Pd truncated hexoctahedral $(\mathrm{THOH})$ shape & One-pot synthesis & $\begin{array}{l}\text { Electrocatalytic performance for } \\
\text { ethanol oxidation }\end{array}$ & Park et al., 2014 \\
\hline Dendritic Bimetallic Platinum-Nickel & One-pot synthesis & Oxygen Reduction Reaction & Eid et al., 2016 \\
\hline Nanocrystals Pd-Ce carbon & Electrospinning technique & Methanol fuel cells & Alvi and Akhtar, 2016 \\
\hline Nanofibers Nickel-Iron Oxide/Carbon Nanofibers & Electrospinning technique & Water oxidation electrocatalysis & Chen et al., 2016 \\
\hline
\end{tabular}


host metal. (b) A geometric effect, when the guest metal atom coordinates with the host metal atom, it undergoes a different atomic arrangement generating new geometry with active sites over the newly generated surface of a bimetallic nanostructure (Siahrostami et al., 2013; Kim et al., 2014). A comparative study between the bimetallic single crystals of nanocatalysts alloy i.e., platinum-nickel and single crystals of pure platinum as well as platinum-carbon fuel cell catalysts in which bimetallic $\mathrm{Pt}_{3} \mathrm{Ni}$ possess different atomic arrangements in the crystalline lattice. The plane with the most tightly packed arrangement of atoms are more active in comparison to conventional and monometallic nanocatalysts. The activity of bimetallic $\mathrm{Pt}_{3} \mathrm{Ni}$ single-crystals increased to 10 and 90 folds with respect to single-crystal Pt and standard Pt/C materials. The theoretical model had predicted that the (111) arrangement decreased the electronic interaction among platinum atoms and oxides at the surface (Service, 2007). The catalytic performance of $\mathrm{Pd}$ can be improved by the rational synthesis of Pd-based BNS (Koenigsmann et al., 2012). Nonnoble metals such as Co (Xu C. et al., 2013; Xu Y. et al., 2013), Cu (Xu et al., 2011), Ni (Maiyalagan and Scott, 2010), Sn (Ding et al., 2013), and Pd based bimetallic electrocatalysts show excellent catalytic activity and are cost effective. In Au-Pd the growth assembly and ligand effects in the nanostructures leads to extremely high activity (Zhang L. et al., 2016). Anisotropic nanostructures with sharp edges are highly reactive and show enhanced catalytic activity. Nanoparticles with an irregular shape lack such properties, resulting in their lower performance. But certain reactions need harsh conditions and pretreatments due to the instability of the morphology and form irregular shapes. The elevated reaction temperature upholds the rate of diffusion in the atoms and strengthens the particles to reorganize. Therefore, it is difficult to study the structural effect unless with a lack of stability in the morphology at high temperature. However, different structures have different properties which are responsible for various catalytic activities. Furthermore, the controlled synthesis of nanomaterials has offered an organized model to clarify the structural effect on the catalytic behavior (He et al., 2010). Wang and co reported that the synthesized bimetallic $\mathrm{Au} / \mathrm{Pd}$ catalysts have a direct correlation between the structures of the catalysts and their catalytic behavior (Wang et al., 2008). Kuai and co have elucidated the excellent electrocatalytic property of Au-Pd alloy in the ORR where polycrystalline Au-Pd alloy showed enhanced catalytic performance compared to the core-shell (Kuai et al., 2012). Table 1 illustrates the diverse application of various BNS in the field of catalysis synthesized adapting different technique.

\section{Bimetallic Nanostructure in the Field of Electrocatalysis Hydrogen Evolution Reaction}

Hydrogen Evolution Reaction (HER) is a half reaction of electrochemical water splitting and requires a proficient and stable electrocatalyst to reduce the overpotential for various applications (Tian et al., 2014; Yang et al., 2016). According to the literature, $\mathrm{Pt} / \mathrm{C}$ is an excellent electrocatalyst for HER, however it is expensive with a low crystal content. An enormous effort has been made for the development of low-cost metal catalysts with phosphides doping (Cui et al., 2015). Ni-Fe phosphide nanorods exhibited electrocatalytic activity with a lesser overpotential of $243 \mathrm{mV}$ (vs. RHE) at $20 \mathrm{~mA} . \mathrm{cm}^{-2}$, smaller Tafel slope of 69.0 $\mathrm{mVdec}^{-1}$, and a higher exchange current density of $79.4 \mu \mathrm{A}$ $\mathrm{cm}^{-2}$ in the HER within basic medium (Du et al., 2018). The synergetic effect developed between $\mathrm{NiP}_{2}$ and $\mathrm{FeP}$ enhanced the electrocatalytic activity of $\mathrm{NiFeP}$ bimetallic phosphides nanorods. Moreover, the anisotropic growth of NiFeP to form rod-like structures also exhibited a vital role for the enhancement of electrocatalytic performance.

$\mathrm{Lu}$ and co demonstrated the dependency over the crystal phases for the development of desired heterometallic nanostructures [i.e., $\mathrm{Au}-\mathrm{Ru}$ nanowires (NWs)]. Ru nanorods can grow epitaxially to $\sim 83.3 \%$ on the $4 \mathrm{H}$ phase of Au NWs while on fcc-twin structures the growth is restricted to $\sim 16.7 \%$ (Lu et al., 2018). Since $\mathrm{Ru}$ is good candidate for HER, a comparative study
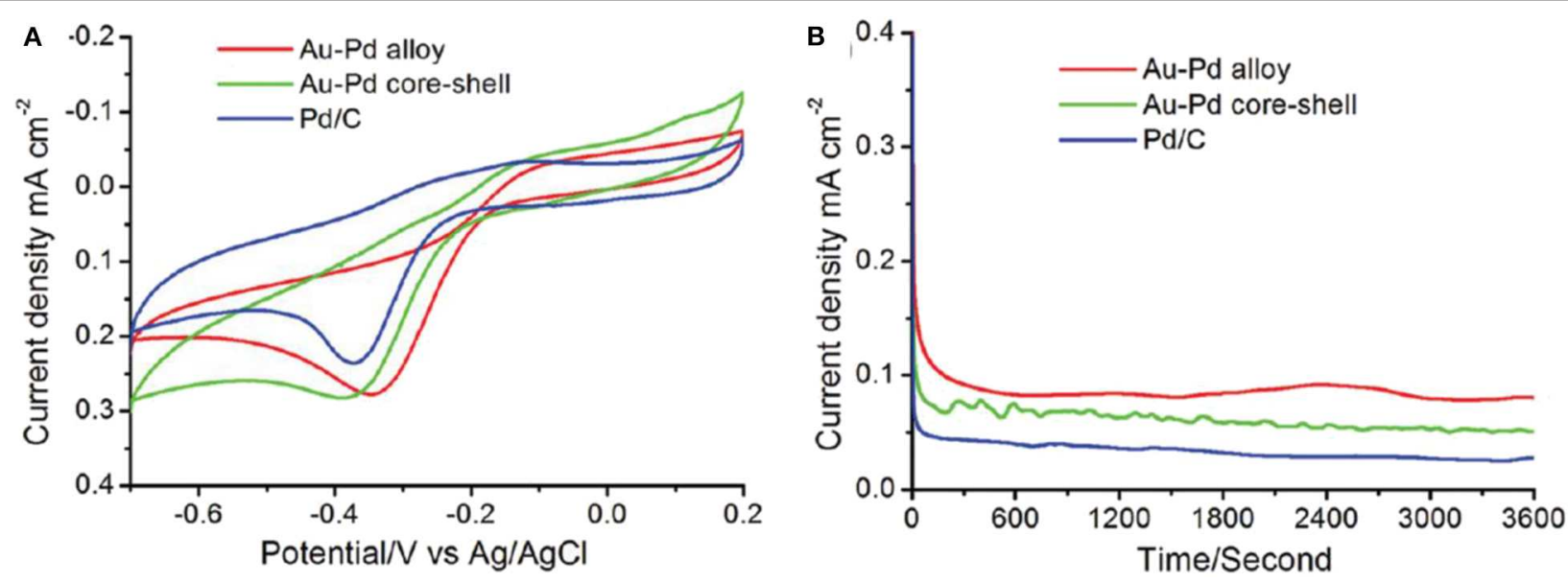

FIGURE 14 | (A) CVs (B) and current-time curves of Au-Pd polycrystalline alloy (red line), core-shell nanostructures (green line), and Pd/C (blue line) electrocatalysts in $\mathrm{O}_{2}$-saturated $1 \mathrm{M} \mathrm{KOH}$ solution measured at room temperature, and the current-time curves are all recorded at the corresponding peak potential [Reprinted with permission from Kuai et al. (2012), Langmuir]. 
TABLE 2 | The mentioned electrocatalysts and their corresponding catalyzing ORR performance [Reprinted with permission from Kuai et al. (2012), Langmuir].

\begin{tabular}{|c|c|c|c|}
\hline Sample & $\begin{array}{l}\text { Peak } \\
\text { potential/V }\end{array}$ & $\begin{array}{c}\text { Peak current } \\
\text { density/ } \\
\mathrm{mA} \mathrm{cm}\end{array}$ & $\begin{array}{c}\text { Stable current } \\
\text { density/ } \\
\mathrm{mA} \mathrm{cm}{ }^{-2}\end{array}$ \\
\hline \multicolumn{4}{|c|}{ Electrocatalytic ORR performance } \\
\hline Au-Pd alloy & -0.342 & 0.281 & 0.080 \\
\hline Au-Pd core- shell & -0.386 & 0.285 & 0.051 \\
\hline $\mathrm{Pd} / \mathrm{C}$ & -0.376 & 0.25 & 0.028 \\
\hline
\end{tabular}

of electrocatalytic activity for HER among Au-Ru NWs, 4H/fcc $\mathrm{Au} \mathrm{NWs}$, and $\mathrm{Ru} / \mathrm{C}$ was carried out. $\mathrm{Au}-\mathrm{Ru} \mathrm{NWs}$ showed the best HER electrocatalytic activity with good stability till 10,000 cycles while $4 \mathrm{H} / \mathrm{fcc} \mathrm{Au}$ NWs exhibited no activity. With the overpotential of $50 \mathrm{mV}, \mathrm{Au}-\mathrm{Ru} \mathrm{NWs}$ attained a current density of $10 \mathrm{~mA} \mathrm{~cm}^{-2}$ which is less in comparison to $\mathrm{Ru} / \mathrm{C}(116 \mathrm{mV})$. In addition to that, it exhibited a lower Tafel slope of $30.8 \mathrm{mV}$ per decade while the $\mathrm{Ru} / \mathrm{C}$ catalyst exhibited $47.8 \mathrm{mV}$ per decade, which suggests $\mathrm{Au}-\mathrm{Ru} \mathrm{NWs}$ holds faster reaction kinetics in HER process. The greater electrocatalytic activity of bimetallic $\mathrm{Au}-\mathrm{Ru} \mathrm{NWs}$ resulted due to the following reasons: (1) One dimensional morphology of $\mathrm{Au}-\mathrm{Ru} \mathrm{NWs}$ facilitates the robust electron transfer throughout HER process. (2) The hierarchical arrangement of $\mathrm{Au}-\mathrm{Ru} \mathrm{NW}$ generates plentiful active sites for HER. The $4 \mathrm{H}$ and fcc-twin morphology of $\mathrm{Ru}$ nanorods possess more surface atoms due to its concave and convex surfaces which provide additional benefits in HER activity (Fan et al., 2016). (3) Due to lattice strain and electron charge transfer between two different metal atoms, there is a change in the electronic band structure generating a favorable electronic band structure for its electrocatalytic performance (Wang et al., 2013; Wang H. et al., 2016).

\section{Oxygen Reduction Reaction}

Pt based bimetallic materials are foremost among all other materials for their superior electrocatalytic behavior in ORR for proton exchange membrane fuel cell (PEMFC) (Choi et al., 2016; O'hayre et al., 2016; Setzler et al., 2016).

A comparative study in the electrocatalytic performance between an Au-Pd alloy and core shell nanostructure in the alkaline medium for ORR has been studied (Kuai et al., 2012). Figure 14A shows cyclic voltammograms (CVs) of an $\mathrm{Au}-\mathrm{Pd}$ alloy (red line), core-shell (green line) nanostructures, and $\mathrm{Pd} / \mathrm{C}$ catalyst (blue line) in an $\mathrm{O}_{2}$-saturated $1 \mathrm{M} \mathrm{KOH}$ solution. The corresponding catalyzed ORR performance is given in Table 2. The Au-Pd alloy nanostructures exhibited the finest electrocatalytic ORR activity. The Au-Pd polycrystalline alloy showed a current density around 1.2 times higher than the $\mathrm{Pd} / \mathrm{C}$ catalyst is shown in Figure 14B. There is a simultaneous decrease in the overpotential of oxygen reduction. The alloy exhibited a positive shift in the peak potential of 44 and $34 \mathrm{mV}$ with respect to the Au-Pd core-shell and Pd/C catalysts, respectively. Hence, the Au-Pd alloy nanostructures showed better ORR performance.

Urchin-like Pt-Ni BNS have shown superior ORR behavior both with and without any anion adsorption electrolytes, due to the synergistic effect developed from alloys. The synthesized Pt-Ni bimetallic showed 20.7 times better specific activity than monometallic Pt (Choi et al., 2016). Pt-Pd carbon supported nanodendrite catalysts were used for ORR performance and were compared with a commercial Pt/C catalyst (Wu et al., 2019a).

A comparative study with three samples of different Pt loading in $\mathrm{PtPd} / \mathrm{C}$ composites and commercial $\mathrm{Pt} / \mathrm{C}$ catalysts of $\mathrm{Pt}$ loading in $\mathrm{Pt}_{1} \mathrm{Pd}_{1} / \mathrm{C}, \mathrm{Pt}_{2} \mathrm{Pd}_{1} / \mathrm{C}, \mathrm{Pt}_{3} \mathrm{Pd}_{1} / \mathrm{C}$, and $\mathrm{Pt} / \mathrm{C}$ were 8.3, 9.5, 12.6, and $24.3 \mu \mathrm{g} . \mathrm{cm}^{-2}$, respectively. The $\mathrm{Pt}_{1} \mathrm{Pd}_{1} / \mathrm{C}$ catalyst showed an excellent performance with good durability. The comparative study of $\mathrm{CV}$ among carbon-supported composites such as $\mathrm{Pt}_{3} \mathrm{Pd}_{1}, \mathrm{Pt}_{2} \mathrm{Pd}_{1}$, and $\mathrm{Pt}_{1} \mathrm{Pd}_{1}$ with the commercial $\mathrm{Pt} / \mathrm{C}$ catalyst is shown in Figure 15A. The CV study was carried out inside $\mathrm{N}_{2}$-purged $0.1 \mathrm{M} \mathrm{HClO}_{4}$ with a scan rate of 50 $\mathrm{mV} / \mathrm{s}$. An ORR polarization curve shows the half-wave potentials of different electrocatalysts were in the sort of commercial $\mathrm{Pt} / \mathrm{C}(863 \mathrm{mV})<\mathrm{Pt}_{3} \mathrm{Pd}_{1} / \mathrm{C}(884 \mathrm{mV})<\mathrm{Pt}_{2} \mathrm{Pd}_{1} / \mathrm{C}(899 \mathrm{mV})<$ $\mathrm{Pt}_{1} \mathrm{Pd}_{1} / \mathrm{C}(916 \mathrm{mV})$ vs. a reversible hydrogen electrode (RHE) shown in Figure 15B. Tafel plots of ORR catalysts have been shown in Figure 15C in which all the catalyst followed similar Tafel slopes in common potential range and followed same reaction mechanism. Figure 15D shows histogram of specific mass activities at $0.9 \mathrm{~V}$ vs. RHE for four catalysts. The $\mathrm{Pt}_{1} \mathrm{Pd}_{1} / \mathrm{C}$ nanodendrite displayed the highest mass activity of $1.16 \mathrm{~A} \mathrm{mg}_{\mathrm{Pt}}^{-1}$ and specific activity of $1.33 \mathrm{~mA} \mathrm{~cm}^{-2}$, which were 7.76 - and 5.32 fold higher compared with the commercial Pt/C catalyst $(0.15 \mathrm{~A}$ $\mathrm{mg}_{\mathrm{Pt}}^{-1}$ and $0.25 \mathrm{~mA} \mathrm{~cm}^{-2}$ ), respectively.

\section{Oxygen Evolution Reaction}

An amorphous $\mathrm{Ni}-\mathrm{Fe}$ oxide/carbon nanofiber compound exhibited a high electrocatalytic response with superior stability in oxygen evolution reaction (OER) using $1 \mathrm{M} \mathrm{KOH}$ electrolyte (Chen et al., 2016). The samples were prepared at different temperatures and studied by measuring the linear sweep voltammetry (LSV) curves that possessed maximum electrocatalytic activity for $\mathrm{Ni}_{1} \mathrm{Fe}_{2}$ material synthesized at $250^{\circ} \mathrm{C}$ and the activity order at different temperatures was as follows: $250>350>450=550^{\circ} \mathrm{C}$. The LSV curve of $\mathrm{Ni}_{1} \mathrm{Fe}_{2}-250$ exhibited a sharp signal due to the oxidation of $\mathrm{Ni}^{2+}$ at $1.45 \mathrm{~V}$ (Trotochaud et al., 2014). When the potential was higher than $1.48 \mathrm{~V}$, there was sudden rise in the oxidation current with the formation of bubbles over the electrode signaling the OER performance preceded. The slight decrease in the electrochemical surface areas (ECSA) of $\mathrm{Ni}_{1} \mathrm{Fe}_{2}-250$ to $\mathrm{Ni}_{1} \mathrm{Fe}_{2}-350$ and the significant decrease occurred subsequently for the samples synthesized at 450 and $550^{\circ} \mathrm{C}$. $\mathrm{Ni}_{1} \mathrm{Fe}_{2}-250$ was the largest ECSA and showed better OER performance, which helps the mass transport.

\section{Bimetallic Nanostructures in the Field of Photocatalysis}

From previous discussion, it was observed that BNS provides novel characteristics by increasing the catalytic performance. In addition to that, photocatalysis has the potential ability to alter the localized surface plasmon resonance (LSPR) to acquire the desired performance. Plasmonic BNS have been extensively studied due to their assimilation of plasmonic function and 

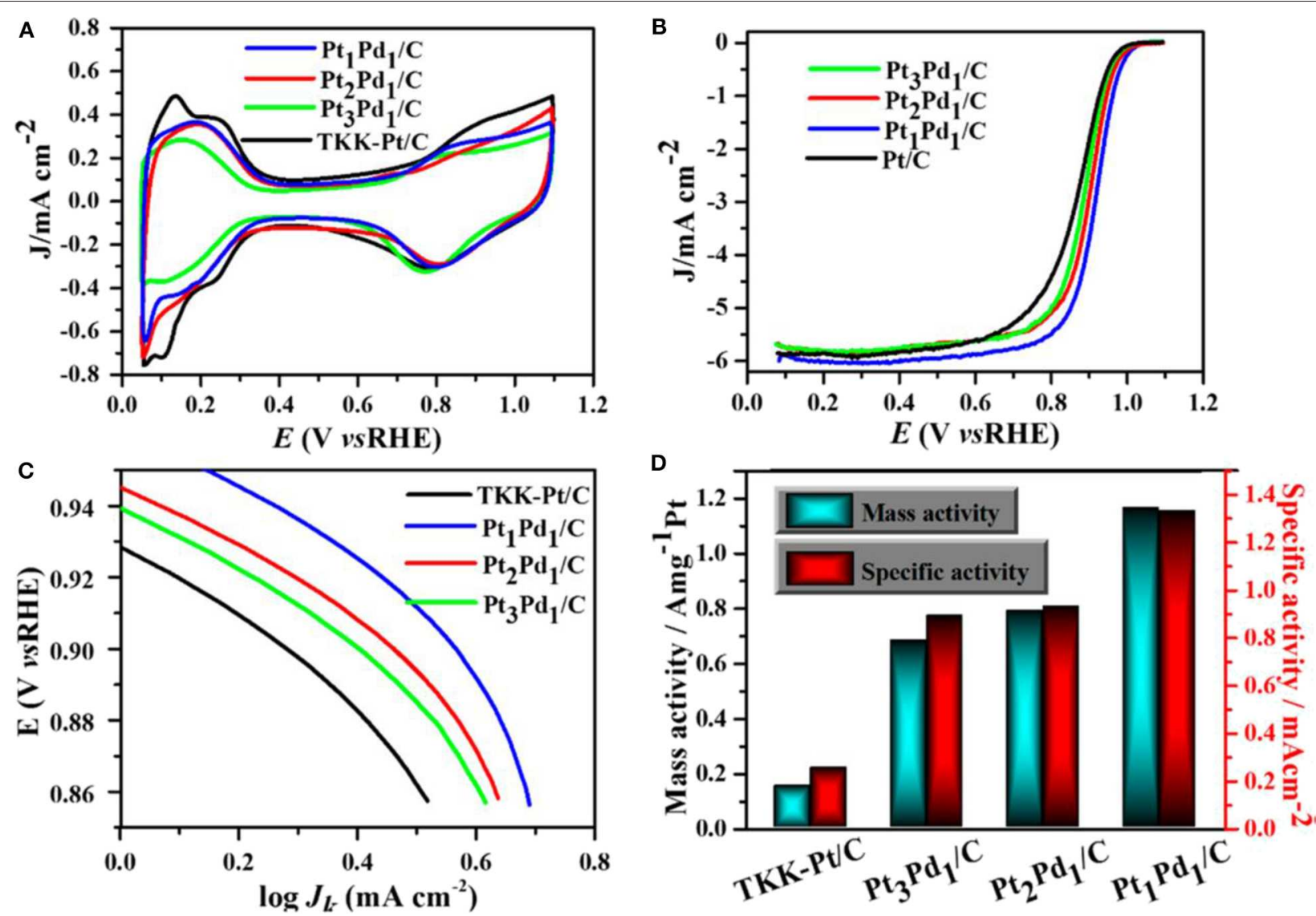

FIGURE 15 | (A) Cyclic voltammograms recorded at room temperature in an $\mathrm{N}_{2}$-purged $0.1 \mathrm{M} \mathrm{HClO}_{4}$ solution with a sweep rate of 50 mV s ${ }^{-1}$. (B) ORR polarization curves recorded in an $\mathrm{O}_{2}$-saturated $0.1 \mathrm{M} \mathrm{HClO}_{4}$ solution with a sweep rate of $10 \mathrm{mV} \mathrm{s}^{-1}$ and a rotation rate of 1,600 rpm. (C) Tafel plots of four kinds of catalysts, and (D) histogram of specific mass activities at 0.9V vs. RHE for four catalysts [Reprinted with permission from Wu et al. (2019b), ACS Sustainable Chemistry and Engineering].

catalytic effect. The massive and continuous demands from the industry are motivating researchers to develop efficient bimetallic photocatalysts. For example, $\mathrm{Au}-\mathrm{Cu}$ triangular nanocrystal was used to catalyze the reduction of 4-nitrophenol to 4-aminophenol and the reaction was carried out under light irradiation as well as in the dark. It was found that the rate constant increased to 32 times when the reaction was conducted in the light in comparison to the dark (Hajfathalian et al., 2015). In addition, Pt-modified $\mathrm{Au}$ nanorods and triangular nanoprisms served as photocatalysts under visible and near-infrared light for hydrogen generation in water-methanol solutions (Zheng et al., 2014; Lou et al., 2016).

Using a modified seeded growth method, Lou and co successfully synthesized three different anisotropic nanostructures: Pt-covered, Pt-edged, and Pt-tipped $\mathrm{Au}$ triangular nanoprisms (TNPs) as shown in Figures 16A-C. Further, the overgrowth of $\mathrm{Pt}$ controlled over the Au surface with the optimal addition of $\mathrm{H}_{2} \mathrm{PtCl}_{6}$ and its photocatalytic dependency over hydrogen generation in water-methanol solutions (2:1 volume ratio) was studied under visible-NIR light irradiation. The rate of generation of $\mathrm{H}_{2}$ decreased in the order of Pt-edged, Pt tipped, and Pt-covered Au TNPs as shown in Figure $16 \mathrm{~F}$. The rate of hydrogen generation in Pt-edged $\mathrm{Au}$
TNPs was $0.167 \mu \mathrm{mol} \mathrm{h}{ }^{-1}$, which was 5 and 3 times greater than Pt-covered $\left(0.031 \mu \mathrm{mol} \mathrm{h}{ }^{-1}\right)$ and Pt-tipped Au TNPs $\left(0.048 \mu \mathrm{mol} \mathrm{h}{ }^{-1}\right)$. The enhancement of $\mathrm{H}_{2}$ generation was well studied from surface plasmon resonance (SPR) in visibleNIR region as shown in Figures 16D,E. The SPR of different TNPs was performed using finite-difference-time-domain (FDTD). TNPs of Au poses different modes of SPR in visibleNIR regions i.e., in-plane dipole surface plasmon resonance (DSPR), multipole surface plasmon resonance (MSPR), and out-of-plane SPR (Tcherniak et al., 2011; Fang et al., 2012). The DSPR and MSPR band of Pt-edged Au TNPs showed red shifted with lesser intensity in comparison to $\mathrm{Au}$ TNPs followed by Pt tipped and Pt-covered Au TNPs, respectively, which confirmed the overgrowth of Pt on Au TNPs. Further, it suggests $\mathrm{Pt}$ is loaded with a stronger electric field and interaction with $\mathrm{Au}$ TNPs in comparison to other anisotropic structures, facilitating more hot electrons transfer among $\mathrm{Au}$ and Pt. Thus, it provides a capable charge separation and $\mathrm{H}_{2}$ generation of Pt-edged Au TNPs. Site-selective silica coating was used to block the overgrowth of the second metal Pd and allowed for preferential deposition at the end or side of synthesized $\mathrm{Au}$ nanorods. $\mathrm{Au} / \mathrm{SiO}_{2} / \mathrm{Pd}$ nanobipyramids showed 


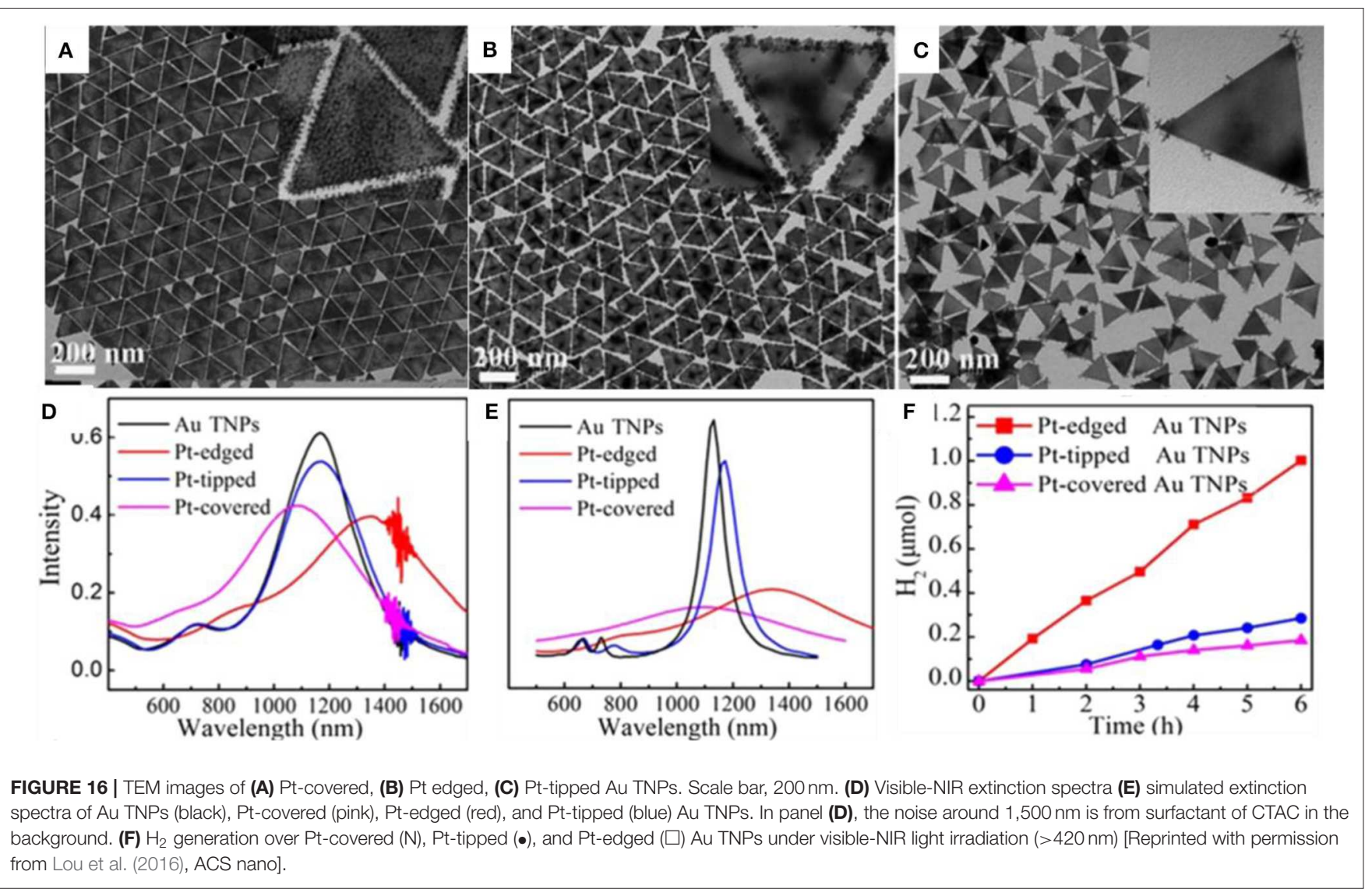

efficient plasmonic photocatalytic performance in the Suzuki coupling reaction.

\section{Oxidation Reaction}

$\mathrm{Rh}, \mathrm{Ag}$, and $\mathrm{Au}$ are well-reported for their catalytic activity, but coupling these three metals is rarely reported. Wei and co grew RhAg on a template of $\mathrm{Au}$ nanorods with different structures and studied their catalytic properties in the oxidation of o-phenylenediamine (OPD) to 2,3-diaminophenazine (DAP). Bimetallic RhAg on Au nanorods with dumbbell-like structures were successfully synthesized (Ye et al., 2014). First, Au nanorods were synthesized and then the synthesized $\mathrm{Au}$ NR solution was mixed with a stabilizing agent $\mathrm{CTAC}, \mathrm{AgNO}_{3}, \mathrm{RhCl}_{3}$, and reducing agent AA. With the same reaction protocol, brushy RhAg-coated Au nanorods and rod-like Au@Ag-Rh nanorattles were synthesized with the additional treatment of different concentrations of $\mathrm{NaOH}$. The addition of $\mathrm{NaOH}$ affects the reaction $\mathrm{pH}$ and, hence, enhances the reducing capability. As a result, an increase in RhAg was observed on the surface of $\mathrm{Au}$ nanorods. Upon the addition of $0.41 \mathrm{mM} \mathrm{NaOH}$, brushy nanorods formed with a length of $52.6 \pm 5.2 \mathrm{~nm}$ and diameter of $29.0 \pm 2.5 \mathrm{~nm}$. With the increase in the concentration of $\mathrm{NaOH}$ to $1.66 \mathrm{mM}$, there was a formation of rod-like nanorattles, the coreshell Au@Ag nanorods covered with porous shell of Rh. The catalytic activity of the three different structures in the oxidation of o-phenylenediamine (OPD) to 2,3-diaminophenazine (DAP) were studied using UV-Vis spectroscopy. DAP has characteristic absorption band at $420 \mathrm{~nm}$. It was found that brushy RhAgcoated gold nanorods showed higher catalytic performance in comparison to dumbbell-like RhAg-tipped gold nanorods and the rod-like Au@Ag-Rh nanorattles. The difference in the catalytic performance is due to the different structures possessing different surface energies and affecting active sites of $\mathrm{Rh}$ in the catalytic activity. Pd Rh nanoframes and nanoboxes have been reported for enhanced catalytic properties compared to the nanocubes (Ye et al., 2015). DAP shows absorption at $420 \mathrm{~nm}$ and OPD does not show any absorption (Cui et al., 2015). On conversion of OPD to DAP, there was a temporary rise in the absorption spectra using bimetallic $\mathrm{Pd}-\mathrm{Rh}$ nanoboxes as the catalyst. Around $95 \%$ of OPD was transformed to DAP after $30 \mathrm{~min}$ with the rise in the absorption at $420 \mathrm{~nm}$. The oxidation of OPDA to DAP using hollow and solid counterparts of nanoframes and nanoboxes have shown enhanced catalytic properties compared to the nanocubes.

\section{SUMMARIES AND OUTLOOK}

In the present review, previous and current evolution of the synthesis technique for anisotropic bimetallic nanomaterials and their applications in catalysis has been discussed. Each synthetic protocol has its own advantage and disadvantage and were designed based on applications that have been 
discussed. Transition or noble bimetallic nanocrystals and their synthesis and the formation of core shell and alloy with anisotropic nanostructures were given emphasis. Designing of the nanostructures and the arrangement of atoms exhibiting a strong relation over the surface of nanocrystals was also discussed. Special attention has been given for controlling the morphology, size, and shape of the nanostructures depending on their application. Additionally, many studies have been discussed, including the amalgamation of multiple elements significantly important for the advanced catalytic behavior (Choi et al., 2016; Du et al., 2018). Electronic arrangement in the BNS being the cause of synergy effect was explained in the various systems. The sequential reduction of gold, silver, palladium, and copper have been discussed in regards to the difference in the reduction potential between two metals that has largely been rationalized in the formation of core shell structures. Rate controlled SMCR reaction offers a strong and adaptable synthetic approach for synthesizing controlled anisotropic multimetallic nanostructures. Au-Pd bimetallic nanocrystals of high-index facets have demonstrated outstanding properties and exhibit their potential for wide applications. Apart from this, noble metal low-cost transition metals have also been given considerable interest for the synthesis of BNS. The GRR has marked a real breakthrough in the advancement of designing and synthesis of bimetallic nanomaterials. GRR was combined with the seedmediated growth and the Kirkendall effect in order to achieve more complex BNS. Noble/non-noble BNS requires harsh reaction environments for one pot technique for the control of nucleation process and the kinetic growth of the two metals. The fact that the one pot technique avoids the prolonged separation technique and the removal of intermediate compounds to

\section{REFERENCES}

Agarwal, S., Greiner, A., and Wendorff, J. H. (2009). Electrospinning of manmade and biopolymer nanofibers-progress in techniques, materials, and applications. Adv. Func. Mater. 19, 2863-2879. doi: 10.1002/adfm.200900591

Ahmad, N., Bon, M., Passerone, D., and Erni, R. (2019). Template assisted in situ synthesis of Ag@Au bimetallic nanostructures employing liquid phase transmission electron microscopy. ACS Nano 13, 13333-13342. doi: 10.1021/acsnano.9b06614

Aika, K., Ban, L. L., Okura, I., Namba, S., and Turkevich, J. (1976). Chemisorption and catalytic activity of a set of platinum catalysts. J. Res. Inst. Cataly. Hokkaido Univ. 24, 54-64.

Alonso, D. M., Wettstein, S. G., and Dumesic, J. A. (2012). Bimetallic catalysts for upgrading of biomass to fuels and chemicals. Chem. Soc. Rev. 41, 8075-8098. doi: $10.1039 / \mathrm{c} 2 \mathrm{cs} 35188 \mathrm{a}$

Alvi, M. A., and Akhtar, M. S. (2016). An effective and low cost PdCe bimetallic decorated carbon nanofibers as electro-catalyst for direct methanol fuel cells applications. J. Alloys Compd. 684, 524-529. doi: 10.1016/j.jallcom.2016.05.101

Armbrster, M., Kovnir, K., Behrens, M., Teschner, D., Grin, Y., and Schlgl, R. (2010). Pd-Ga intermetallic compounds as highly selective semihydrogenation catalysts. J. Am.Chem. Soc. 132, 14745-14747. doi: 10.1021/ja106568t

Ataee-Esfahani, H., Wang, L., Nemoto, Y., and Yamauchi, Y. (2010). Synthesis of bimetallic Au@ Pt nanoparticles with $\mathrm{Au}$ core and nanostructured Pt shell toward highly active electrocatalysts. Chem. Mater. 22, 6310-6318. doi: 10.1021/cm102074w

Banaee, M. G., and Crozier, K. B. (2010). Au nanorings as substrates for surface-enhanced Raman scattering. Optics Lett. 35, 760-762. doi: 10.1364/OL.35.000760 save time with a higher yield was explained with examples. BNS synthesized using hydrothermal protocol offers high purity, controlled morphology, uniformity, and fewer defects. Different temperatures in the hydrothermal technique provides diverse structures. The electrospinning technique is used for achieving extremely thin fibers of a few nanometers with high surface areas and pronounced physiochemical characteristics. In addition to morphology, the change in the precursor ratio in the mother solution exhibits wide tunability in the application. The synergy effect between two distinct atoms on a nanocrystal surface has been discussed widely with regards to their excellent catalytic activity, greater stability, and higher selectivity compared to monometallic analogs. ORR, HER, OER, and other catalysts have been tremendously accelerated by the BNS. Platinum, palladium monometallic, and bimetallic Au-Pd polycrystalline show higher electrocatalytic performance than $\mathrm{Pd} / \mathrm{C}$. Ni-Fe phosphides nanorods show better electrocatalytic performance with a smaller overpotential in comparison to $\mathrm{Pt} / \mathrm{C}$ for HER.

\section{AUTHOR CONTRIBUTIONS}

$\mathrm{PB}$ and $\mathrm{MB}$ prepared the detailed synthesis part of bimetallic nanostructures. SS prepared the application in catalysis. MS and AS helped in the required modification and helpful discussion in this review.

\section{ACKNOWLEDGMENTS}

$\mathrm{PB}$ and $\mathrm{AS}$ are grateful to the SERB, New Delhi, India for funding to conduct the research (CRG/2018/003533).

Barakat, N. A., Abadir, M. F., Akhtar, M. S., El-Newehy, M., Shin, Y. S., and Kim, H. Y. (2012). Synthesis and characterization of Pd-doped Co nanofibers as a multifunctional nanostructure. Mater. Lett. 85, 120-123. doi: 10.1016/j.matlet.2012.06.099

Barakat, N. A., Khalil, K. A., Mahmoud, I. H., Kanjwal, M. A., Sheikh, F. A., and Kim, H. Y. (2010). CoNi bimetallic nanofibers by electrospinning: nickel-based soft magnetic material with improved magnetic properties. J. Phys. Chem.C 114, 15589-15593. doi: 10.1021/jp1041074

Bhardwaj, N., and Kundu, S. C. (2010). Electrospinning: a fascinating fiber fabrication technique. Biotechnol. Adv. 28, 325-347. doi: 10.1016/j.biotechadv.2010.01.004

Bradley, J. S., Hill, E. W., Klein, C., Chaudret, B., and Duteil, A. (1993). Synthesis of monodispersed bimetallic palladium-copper nanoscale colloids. Chem. Mater. 5, 254-256. doi: 10.1021/cm00027a005

Byrappa, K., and Adschiri, T. (2007). Hydrothermal technology for nanotechnology. Prog. Cryst. Growth Charact. Mater. 53, 117-166. doi: 10.1016/j.pcrysgrow.2007.04.001

Chen, H., Huang, X., Zhou, L. J., Li, G. D., Fan, M., and Zou, X. (2016). Electrospinning synthesis of bimetallic nickel-iron oxide/carbon composite nanofibers for efficient water oxidation electrocatalysis. Chem. Cat. Chem. 8, 992-1000. doi: 10.1002/cctc.201501326

Chen, S., Su, H., Wang, Y., Wu, W., and Zeng, J. (2015). Size-controlled synthesis of platinum-copper hierarchical trigonal bipyramid nanoframes. Angew. Chem. Int. Ed. 54, 108-113. doi: 10.1002/anie.201408399

Choi, J.-P., Fields-Zinna, C. A., Stiles, R. L., Balasubramanian, R., Douglas, A. D., Crowe, M. C., et al. (2010). Reactivity of $\left[\mathrm{Au}_{25}\left(\mathrm{SCH}_{2} \mathrm{CH}_{2} \mathrm{Ph}\right)\right.$ 18] 1- nanoparticles with metal ions. J. Phys. Chem. C 114, 15890-15896. doi: 10.1021/jp9101114 
Choi, K. H., Jang, Y., Chung, D. Y., Seo, P., Jun, S. W., Lee, J. E., et al. (2016). A simple synthesis of urchin-like Pt-Ni bimetallic nanostructures as enhanced electrocatalysts for the oxygen reduction reaction. Chem. Commun. 52, 597-600. doi: 10.1039/c5cc08088f

Corpuz, R. D., Ishida, Y., Nguyen, M. T., and Yonezawa, T. (2017). Synthesis of positively charged photoluminescent bimetallic Au-Ag nanoclusters by double-target sputtering method on a biocompatible polymer matrix. Langmuir 33, 9144-9150. doi: 10.1021/acs.langmuir.7b02011

Cui, W., Liu, Q., Xing, Z., Asiri, A. M., Alamry, K. A., and Sun, X. (2015). MoP nanosheets supported on biomass-derived carbon flake: Onestep facile preparation and application as a novel high-active electrocatalyst toward hydrogen evolution reaction. Appl. Catal. B Environ. 164, 144-150. doi: 10.1016/j.apcatb.2014.09.016

Da Silva, A. G., Rodrigues, T. S., Haigh, S. J., and Camargo, P. H. (2017). Galvanic replacement reaction: recent developments for engineering metal nanostructures towards catalytic applications. Chem. Commun. 53, 7135-7148. doi: $10.1039 / \mathrm{c} 7 \mathrm{cc} 02352 \mathrm{a}$

Demir, M. M., Gulgun, M. A., Menceloglu, Y. Z., Erman, B., Abramchuk, S. S., Makhaeva, E. E., et al. (2004). Palladium nanoparticles by electrospinning from poly (acrylonitrile-co-acrylic acid)- $\mathrm{PdCl}_{2}$ solutions. relations between preparation conditions, particle size, and catalytic activity. Macromolecules 37, 1787-1792. doi: 10.1021/ma035163x

Ding, L. X., Wang, A. L., Ou, Y. N., Li, Q., Guo, R., Zhao, W. X., et al. (2013). Hierarchical Pd-Sn alloy nanosheet dendrites: an economical and highly active catalyst for ethanol electrooxidation. Sci. Rep. 3:1181. doi: 10.1038/srep01181

Du, Y., Li, Z., Liu, Y., Yang, Y., and Wang, L. (2018). Nickel-iron phosphides nanorods derived from bimetallic-organic frameworks for hydrogen evolution reaction. Appl. Surf. Sci. 457, 1081-1086. doi: 10.1016/j.apsusc.2018.06.167

Eid, K., Wang, H., Malgras, V., Alothman, Z. A., Yamauchi, Y., and Wang, L. (2016). Facile synthesis of porous dendritic bimetallic platinum-nickel nanocrystals as efficient catalysts for the oxygen reduction reaction. Chem. Asian J. 11, 1388-1393. doi: 10.1002/asia.201600055

Esumi, K., Tano, T., Torigoe, K., and Meguro, K. (1990). Preparation and characterization of bimetallic palladium-copper colloids by thermal decomposition of their acetate compounds in organic solvents. Chem. Mater. 2, 564-567.doi: 10.1021/cm00011a019

Fan, Z., Luo, Z., Huang, X., Li, B., Chen, Y., Wang, J., et al. (2016). Synthesis of $4 \mathrm{H} / \mathrm{fcc}$ noble multimetallic nanoribbons for electrocatalytic hydrogen evolution reaction. J. Am. Chem. Soc. 138, 1414-1419. doi: $10.1021 /$ jacs.5b12715

Fang, Y., Chang, W. S., Willingham, B., Swanglap, P., Dominguez-Medina, S., and Link, S. (2012). Plasmon emission quantum yield of single gold nanorods as a function of aspect ratio. Acs Nano 6, 7177-7184. doi: 10.1021/nn3022469

Faraday, M. (1857). The bakerian lecture-experimental relations of gold (and other metals) to light. Philos. T. R. Soc. B 147, 145-181. doi: 10.1098/rstl.1857.0011

Focaccia, M., and Simili, R. (2007). "Luigi Galvani, physician, surgeon, physicist: from animal electricity to electro-physiology," in Brain, Mind and Medicine: Essays in Eighteenth-Century Neuroscience (London: Springer), 145-158.

Gan, Z., Xia, N., and Wu, Z. (2018). Discovery, mechanism, and application ofantigalvanic reaction. Acc. Chem. Res. 51, 2774-2783. doi: 10.1021/acs.accounts.8b00374

Ghouri, Z. K., Barakat, N. A., Park, M., Kim, B. S., and Kim, H. Y. (2015). Synthesis and characterization of $\mathrm{Co} / \mathrm{SrCO}_{3}$ nanorods-decorated carbon nanofibers as novel electrocatalyst for methanol oxidation in alkaline medium. Ceram. Int. 41, 6575-6582. doi: 10.1016/j.ceramint.2015.01.103

Gołabiewska, A., Lisowski, W., Jarek, M., Nowaczyk, G., Michalska, M., Jurga, S., et al. (2017). The effect of metals content on the photocatalytic activity of $\mathrm{TiO}_{2}$ modified by $\mathrm{Pt} / \mathrm{Au}$ bimetallic nanoparticles prepared by sol-gel method. Mol. Catal. 442, 154-163. doi: 10.1016/j.mcat.2017.09.004

González, E., Arbiol, J., and Puntes, V. F. (2011). Carving at the nanoscale: sequential galvanic exchange and Kirkendall growth at room temperature. Science 334, 1377-1380. doi: 10.1126/science.1212822

Hajfathalian, M., Gilroy, K. D., Yaghoubzade, A., Sundar, A., Tan, T., Hughes, R. A., et al. (2015). Photocatalytic enhancements to the reduction of 4-nitrophenol by resonantly excited triangular gold-copper nanostructures. J. Phys. Chem. C 119, 17308-17315. doi: 10.1021/acs.jpcc.5b04618

Harada, M., Asakura, K., Ueki, Y., and Toshima, N. (1992). Structure of polymer-protected palladium-platinum bimetallic clusters at the oxidized state: extended x-ray absorption fine structure analysis. J. Phys. Chem. 96, 9730-9738. doi: 10.1021/j100203a030

He, W., Wu, X., Liu, J., Hu, X., Zhang, K., Hou, S., et al. (2010). Design of AgM bimetallic alloy nanostructures $(\mathrm{M}=\mathrm{Au}, \mathrm{Pd}, \mathrm{Pt})$ with tunable morphology and peroxidase-like activity. Chem. Mater. 22, 2988-2994. doi: 10.1021/cm100393v

Henglein, A. (1993). Physicochemical properties of small metal particles in solution: "microelectrode" reactions, chemisorption, composite metal particles, and the atom-to-metal transition. J. Phys. Chem. 97, 5457-5471. doi: 10.1021/j100123a004

Huang, X., Zhang, H., Guo, C., Zhou, Z., and Zheng, N. (2009). Simplifying the creation of hollow metallic nanostructures: one-pot synthesis of hollow palladium/platinum single-crystalline nanocubes. Angew Chem. Int. Ed. Engl. 48, 4808-4812. doi: 10.1002/anie.200900199

Kim, D., Resasco, J., Yu, Y., Asiri, A. M., and Yang, P. (2014). Synergistic geometric and electronic effects for electrochemical reduction of carbon dioxide using gold-copper bimetallic nanoparticles. Nat. Commun. 5:4948. doi: $10.1038 /$ ncomms5948

Koenigsmann, C., Scofield, M. E., Liu, H., and Wong, S. S. (2012). Designing enhanced one-dimensional electrocatalysts for the oxygen reduction reaction: Probing size-and composition-dependent electrocatalytic behavior in noble metal nanowires. J. Phys. Chem. Lett. 3, 3385-3398. doi: 10.1021/jz301457h

Kuai, L., Yu, X., Wang, S., Sang, Y., and Geng, B. (2012). Au-Pd alloy and core-shell nanostructures: One-pot coreduction preparation, formation mechanism, and electrochemical properties. Langmuir 28, 7168-7173. doi: 10.1021/la300813z

Kunz, M. R., Mcclain, S. M., Chen, D. P., Koczkur, K. M., Weiner, R. G., and Skrabalak, S. E. (2017). Seed-mediated co-reduction in a large lattice mismatch system: synthesis of Pd-Cu nanostructures. Nanoscale 9, 7570-7576. doi: $10.1039 / \mathrm{c} 7 \mathrm{nr} 02918 \mathrm{~g}$

Lee, Y. W., Kim, D., Hong, J. W., Kang, S. W., Lee, S. B., and Han, S. W. (2013). Kinetically controlled growth of polyhedral bimetallic alloy nanocrystals exclusively bound by high-index facets: Au-Pd hexoctahedra. Small 9, 660-665.doi: 10.1002/smll.201201813

Lim, B., Kobayashi, H., Yu, T., Wang, J., Kim, M. J., Li, Z.-Y., et al. (2010). Synthesis of Pd-Au bimetallic nanocrystals via controlled overgrowth. J. Am. Chem. Soc. 132, 2506-2507. doi: 10.1021/ja909787h

Liu, M., Zheng, Y., Xie, S., Li, N., Lu, N., Wang, J., et al. (2013). Facile synthesis of Pd-Ir bimetallic octapods and nanocages through galvanic replacement and co-reduction, and their use for hydrazine decomposition. Phys. Chem. Chem. Phys. 15, 11822-11829. doi: 10.1039/c3cp51950c

Liu, X., and Astruc, D. (2017). From galvanic to anti-galvanic synthesis of bimetallic nanoparticles and applications in catalysis, sensing, and materials science. Adv. Mater. 29:1605305. doi: 10.1002/adma.201605305

Lou, Z., Fujitsuka, M., and Majima, T. (2016). Pt-Au triangular nanoprisms with strong dipole plasmon resonance for hydrogen generation studied by singleparticle spectroscopy. ACS Nano 10, 6299-6305. doi: 10.1021/acsnano.6b02494

Lu, N., Wang, J., Xie, S., Xia, Y., and Kim, M. J. (2013). Enhanced shape stability of $\mathrm{Pd}-\mathrm{Rh}$ core-frame nanocubes at elevated temperature: in situ heating transmission electronmicroscopy. Chem. Commun. 49, 11806-11808. doi: 10.1039/C3CC46465B

Lu, Q., Wang, A.-L., Gong, Y., Hao, W., Cheng, H., Chen, J., et al. (2018). Crystal phase-based epitaxial growth of hybrid noble metal nanostructures on $4 \mathrm{H} / \mathrm{fcc}$ Au nanowires. Nat. Chem. 10, 456-461. doi: 10.1038/s41557-018-0012-0

Lu, X., Tuan, H. Y., Chen, J., Li, Z. Y., Korgel, B. A., and Xia, Y. (2007). Mechanistic studies on the galvanic replacement reaction between multiply twinned particles of $\mathrm{Ag}$ and $\mathrm{HAuCl}_{4}$ in an organic medium. J. Am. Chem. Soc. 129, 1733-1742. doi: 10.1021/ja067800f

Mahmoud, M. A., and El-Sayed, M. A. (2014). Enhancing catalytic efficiency of hollow palladium nanoparticles by photothermal heating of gold nanoparticles added to the cavity: palladium-gold nanorattles. Chem.Cat. Chem. 6, 3540-3546. doi: 10.1002/cctc.201402644

Mahmoud, M. A., Narayanan, R., and El-Sayed, M. A. (2013). Enhancing colloidal metallic nanocatalysis: sharp edges and corners for solid nanoparticles and cage effect for hollow ones. Acc. Chem. Res. 46, 1795-1805. doi: 10.1021/ar3002359

Maiyalagan, T., and Scott, K. (2010). Performance of carbon nanofiber supported $\mathrm{Pd}-\mathrm{Ni}$ catalysts for electro-oxidation of ethanol in alkaline medium. J. Power Sources 195, 5246-5251. doi: 10.1016/j.jpowsour.2010.03.022

Mallikarjuna, K., Reddy, Y. V. M., Sravani, B., Madhavi, G., Kim, H., Agarwal, S., et al. (2018). Simple synthesis of biogenic PdAg bimetallic nanostructures for an 
ultra-sensitive electrochemical sensor for sensitive determination of uric acid. J. Electroanal. Chem. 822, 163-170. doi: 10.1016/j.jelechem.2018.05.019

Meitzner, G., Via, G., Lytle, F., and Sinfelt, J. (1983). Structure of bimetallic clusters. Extended x-ray absorption fine structure (EXAFS) studies of Ir-Rh clusters. J. Chem. Phys. 78, 2533-2541. doi: 10.1063/1.445006

Meitzner, G., Via, G., Lytle, F., and Sinfelt, J. (1985). Structure of bimetallic clusters. Extended x-ray absorption fine structure (EXAFS) studies of $\mathrm{Ag}-\mathrm{Cu}$ and $\mathrm{Au}-\mathrm{Cu}$ clusters. J. Chem. Phys. 83, 4793-4799. doi: 10.1063/1.449005

Mizukoshi, Y., Okitsu, K., Maeda, Y., Yamamoto, T. A., Oshima, R., and Nagata, Y. (1997). Sonochemical preparation of bimetallic nanoparticles of gold/palladium in aqueous solution. J. Phys. Chem. B 101, 7033-7037. doi: $10.1021 /$ jp 9638090

Nafria, R., Gen, A., Ibez, M., Arbiol, J., de la Ram-Rez Piscina, P., Homs, N., et al. (2016). Co-Cu nanoparticles: synthesis by galvanic replacement and phase rearrangement during catalytic activation. Langmuir 32, 2267-2276. doi: 10.1021/acs.langmuir.5b04622

O'hayre, R., Cha, S. W., Colella, W., and Prinz, F. B. (2016). Fuel Cell Fundamentals. New York, NY: John Wiley and Sons.

Ovais, M., Raza, A., Naz, S., Islam, N. U., Khalil, A. T., Ali, S., et al. (2017). Current state and prospects of the phytosynthesized colloidal gold nanoparticles and their applications in cancer theranostics. Appl. Microbiol. Biotechnol. 101, 3551-3565. doi: 10.1007/s00253-017-8250-4

Ozel, T., Ashley, M. J., Bourret, G. R., Ross, M. B., Schatz, G. C., and Mirkin, C. A. (2015). Solution-dispersible metal nanorings with deliberately controllable compositions and architectural parameters for tunable plasmonic response. Nano Lett. 15, 5273-5278. doi: 10.1021/acs.nanolett.5b01594

Park, Y., Lee, Y. W., Kang, S. W., and Han, S. W. (2014). One-pot synthesis of Au@Pd core-shell nanocrystals with multiple high-and low-index facets and their high electrocatalytic performance. Nanoscale 6, 9798-9805. doi: $10.1039 / \mathrm{c} 4 \mathrm{nr} 02034 \mathrm{k}$

Paul, A. (2004). The Kirkendall effect in solid state diffusion. Laboratory of Materials and Interface Chemistry. doi: 10.6100/IR579448

Piccolo, L. (2012). "Surface studies of catalysis by metals: nanosize and alloying effects," in Nanoalloys (Boston, MA: Springer), 369-404. doi: 10.1007/978-1-4471-4014-6_11

Prevo, B. G., Esakoff, S. A., Mikhailovsky, A., and Zasadzinski, J. A. (2008). Scalable routes to gold nanoshells with tunable sizes and response to near-infrared pulsed-laser irradiation. Small 4, 1183-1195. doi: 10.1002/smll.200701290

Rechberger, F., and Niederberger, M. (2017). Synthesis of aerogels: from molecular routes to 3-dimensional nanoparticle assembly. Nanoscale Horiz. 2, 6-30. doi: $10.1039 / \mathrm{C} 6 \mathrm{NH} 00077 \mathrm{~K}$

Reguera, J., de Aberasturi, D. J., Henriksen-Lacey, M., Langer, J., Espinosa, A., Szczupak, B., et al. (2017). Janus plasmonic-magnetic gold-iron oxide nanoparticles as contrast agents for multimodal imaging, Nanoscale 9, 9467-9480. doi: 10.1039/C7NR01406F

Remita, S., Mostafavi, M., and Delcourt, M. (1996). Bimetallic Ag-Pt and AuPt aggregates synthesized by radiolysis. Radiat. Phys. Chem. 47, 275-279. doi: 10.1016/0969-806X(94)00172-G

Ruditskiy, A., Peng, H.-C., and Xia, Y. (2016). Shape-controlled metal nanocrystals for heterogeneous catalysis. Ann. Rev. Chem. Biomol. Eng. 7, 327-348. doi: 10.1146/annurev-chembioeng-080615-034503

Sahu, P., and Prasad, B. (2015). Preparation of Ag (Shell)-Au (Core) nanoparticles by anti-Galvanic reactions: are capping agents the "real heroes" of reduction? Colloid Surface A 478, 30-35. doi: 10.1016/j.colsurfa.2015.03.028

Sajanlal, P. R., Sreeprasad, T. S., Samal, A. K., and Pradeep, T. (2011). Anisotropic nanomaterials: structure, growth, assembly, and functions. Nano Rev. 2:5883. doi: 10.3402/nano.v2i0.5883

Saleem, F., Zhang, Z., Cui, X., Gong, Y., Chen, B., Lai, Z., et al. (2019). Elemental segregation in multimetallic core-shell nanoplates. J. Am. Chem. Soc. 141, 14496-14500. doi: 10.1021/jacs.9b05197

Santana, J. S., Koczkur, K. M., and Skrabalak, S. E. (2018). Kinetically controlled synthesis of bimetallic nanostructures by flowrate manipulation in a continuous flow droplet reactor. Reac. Chem. Eng. 3, 437-441. doi: 10.1039/c8re00077h

Schmidt, T., Noeske, M., Gasteiger, H. A., Behm, R., Britz, P., Brijoux, W., et al. (1997). Electrocatalytic activity of PtRu alloy colloids for $\mathrm{CO}$ and $\mathrm{CO} / \mathrm{H}_{2}$ electrooxidation: stripping voltammetry and rotating disk measurements. Langmuir 13, 2591-2595.doi: 10.1021/la962068r
Senthilkumar, N., Gnana Kumar, G., and Manthiram, A. (2018). 3D hierarchical core-shell nanostructured arrays on carbon fibers as catalysts for direct urea fuel cells. Adv. Energ. Mater. 8:1702207. doi: 10.1002/aenm.201702207

Service R. F. (2007) Chemistry. Platinum in fuel cells gets a helping hand. Science 315:172. doi: $10.1126 /$ science.315.5809.172

Setzler, B. P., Zhuang, Z., Wittkopf, J. A., and Yan, Y. (2016). Activity targets for nanostructured platinum-group-metal-free catalysts in hydroxide exchange membrane fuel cells. Nat. Nanotechnol. 11, 1020-1025. doi: 10.1038/NNANO.2016.265

Sharma, G., Kumar, A., Sharma, S., Naushad, M., Dwivedi, R.P. ALOthman, Z. A., et al. (2017). Novel development of nanoparticles to bimetallic nanoparticles and their composite: a review. J. King Saud Univ. Sci. 31:12. doi: 10.1016/j.jksus.2017.06.012

Siahrostami, S., Verdaguer-Casadevall, A., Karamad, M., Deiana, D., Malacrida, P., Wickman, B., et al. (2013). Enabling direct $\mathrm{H}_{2} \mathrm{O}_{2}$ production through rational electrocatalyst design. Nat. Mater. 12:1137. doi: 10.1038/NMAT3795

Sinfelt, J., Via, G., and Lytle, F. (1980). Structure of bimetallic clusters. Extended xray absorption fine structure (EXAFS) studies of Ru-Cu clusters. J. Chem. Phys. 72, 4832-4844. doi: 10.1063/1.439821

Sinfelt, J., Via, G., and Lytle, F. (1982). Structure of bimetallic clusters. Extended xray absorption fine structure (EXAFS) studies of Pt-Ir clusters. J. Chem. Phys. 76, 2779-2789. doi: 10.1063/1.443380

Sinfelt, J., Via, G., Lytle, F., and Greegor, R. (1981). Structure of bimetallic clusters. extended $x$-ray absorption fine structure (EXAFS) studies of Os-Cu clusters. J. Chem. Phys. 75, 5527-5537.doi: 10.1063/1.441957

Strand, M. B., Leong, G. J., Tassone, C. J., Larsen, B., Neyerlin, K., Gorman, B., et al. (2016). Mechanistic study of shape-anisotropic nanomaterials synthesized via spontaneous galvanic displacement. J. Phys. Chem. C 120, 25053-25060. doi: 10.1021/acs.jpcc.6b07363

Straney, P. J., Marbella, L. E., Andolina, C. M., Nuhfer, N. T., and Millstone, J. E. (2014). Decoupling mechanisms of platinum deposition on colloidal gold nanoparticle substrates. J. Am. Chem. Soc. 136, 7873-7876. doi: $10.1021 / j a 504294 \mathrm{p}$

Sun, J., Wu, H., and Jin, Y. (2014). Synthesis of thiolated $\mathrm{Ag} / \mathrm{Au}$ bimetallic nanoclusters exhibiting an anti-galvanic reduction mechanism and composition-dependent fluorescence. Nanoscale 6, 5449-5457. doi: $10.1039 / \mathrm{c} 0 \mathrm{xx} 00000 \mathrm{x}$

Sun, L., Zhang, Q., Li, G. G., Villarreal, E., Fu, X., and Wang, H. (2017) Multifaceted gold-palladium bimetallic nanorods and their geometric, compositional, and catalytic tunabilities. ACS Nano 11, 3213-3228. doi: 10.1021/acsnano.7b00264

Sun, Y., and Xia, Y. (2002). Shape-controlled synthesis of gold and silver nanoparticles. Science 298, 2176-2179. doi: 10.1126/science.1077229

Sun, Y., and Xia, Y. (2004). Mechanistic study on the replacement reaction between Ag nanostructures and chloroauric acid in aqueous medium. J. Am. Chem. Soc. 126, 3892-3901. doi: 10.1021/ja039734c

Tao, A. R., Habas, S., and Yang, P. (2008). Shape control of colloidal metal nanocrystals. Small 4, 310-325. doi: 10.1002/smll.200701295

Tao, F. F. (2012). Synthesis, catalysis, surface chemistry and structure of bimetallic nanocatalysts. Chem. Soc. Rev. 41, 7977-7979. doi: 10.1039/c2cs90093a

Tao, F. F., Nguyen, L., Zhang, S., Li, Y., Tang, Y., Zhang, L., et al. (2016). Formation of second-generation nanoclusters on metal nanoparticles driven by reactant gases. Nano Lett. 16, 5001-5009. doi: 10.1021/acs.nanolett.6b01718

Tcherniak, A., Dominguez-Medina, S., Chang, W. S., Swanglap, P., Slaughter, L. S., Landes, C. F., et al. (2011). One-photon plasmon luminescence and its application to correlation spectroscopy as a probe for rotational and translational dynamics of gold nanorods. J. Phys. Chem. C 115, 15938-15949. doi: $10.1021 /$ jp206203s

Tian, J., Liu, Q., Cheng, N., Asiri, A. M., and Sun, X. (2014). Self-supported $\mathrm{Cu} 3 \mathrm{P}$ nanowire arrays as an integrated high-performance three-dimensional cathode for generating hydrogen from water. Angew. Chem. Int. Ed. Engl. 53, 9577-9581. doi: 10.1002/anie.201403842

Toshima, N., and Wang, Y. (1993). Novel preparation, characterization and catalytic properties of polymer-protected $\mathrm{Cu} / \mathrm{Pd}$ bimetallic colloid. Chem. Lett. 22, 1611-1614. doi: 10.1246/cl.1993.1611

Toshima, N., and Wang, Y. (1994a). Polymer-protected cu/pd bimetallic clusters. Adv. Mater. 6, 245-247. doi: 10.1002/adma.19940060316 
Toshima, N., and Wang, Y. (1994b). Preparation and catalysis of novel colloidal dispersions of copper/noble metal bimetallic clusters. Langmuir 10, 4574-4580. doi: 10.1021/la00024a031

Trotochaud, L., Young, S. L., Ranney, J. K., and Boettcher, S. W. (2014). Nickeliron oxyhydroxide oxygen-evolution electrocatalysts: the role of intentional and incidental iron incorporation. J. Am. Chem. Soc. 136, 6744-6753. doi: $10.1021 /$ ja $502379 \mathrm{c}$

Turkevich, J., and Kim, G. (1970). Palladium: preparation and catalytic properties of particles of uniform size. Science 169, 873-879. doi: 10.1126/science.169.3948.873

Turkevich, J., Stevenson, P. C., and Hillier, J. (1951). A study and nucleation and growth processes in the synthesis of colloidal gold. Discuss. Faraday Soc. 11, 55-75. doi: 10.1039/DF9511100055

Vilana, J., Lorenzo, M., Gomez, E., and Vallés, E. (2015). Electrochemical deposition of CoNi micro/nanostructures as new materials for electrochemical sensing of glucose. Mater. Lett. 159, 154-158. doi: 10.1016/j.matlet.2015.06.116

Wang, D., Villa, A., Porta, F., Prati, L., and Su, D. (2008). Bimetallic gold/palladium catalysts: Correlation between nanostructure and synergistic effects. J. Phys. Chem. C 112, 8617-8622. doi: 10.1021/jp800805e

Wang, D., Xin, H. L., Hovden, R., Wang, H., Yu, Y., Muller, D. A., et al. (2013). Structurally ordered intermetallic platinum-cobalt core-shell nanoparticles with enhanced activity and stability as oxygen reduction electrocatalysts. Nat. Mater. 12, 81-87. doi: 10.1038/nmat3458

Wang, H., Xu, S., Tsai, C., Li, Y., Liu, C., Zhao, J., et al. (2016). Direct and continuous strain control of catalysts with tunable battery electrode materials. Science 354, 1031-1036. doi: 10.1126/science.aaf7680

Wang, L., Nemoto, Y., and Yamauchi, Y. (2011). Direct synthesis of spatiallycontrolled Pt-on-Pd bimetallic nanodendrites with superior electrocatalytic activity. J. Am. Chem. Soc. 133, 9674-9677. doi: 10.1021/ja202655j

Wang, L., Wang, H., Nemoto, Y., and Yamauchi, Y. (2010). Rapid and efficient synthesis of platinum nanodendrites with high surface area by chemical reduction with formic acid. Chem. Mater. 22, 2835-2841. doi: $10.1021 / \mathrm{cm} 9038889$

Wang, M., Wu, Z., Chu, Z., Yang, J., and Yao, C. (2014). Chemicophysical synthesis of surfactant-and ligand-free gold nanoparticles and their anti-galvanic reduction property. Chem. Asian J. 9, 1006-1010. doi: 10.1002/asia.201301562

Wang, W., Yan, Y., Zhou, N., Zhang, H., Li, D., and Yang, D. (2016). Seedmediated growth of Au nanorings with size control on Pd ultrathin nanosheets and their tunable surface plasmonic properties. Nanoscale 8, 3704-3710. doi: 10.1039/C5NR08613B

Wu, R., Li, Y., Gong, W., and Shen, P. K. (2019a). One-pot synthesis of Pt-Pd bimetallic nanodendrites with enhanced electrocatalytic activity for oxygen reduction reaction. ACS Sus. Chem. Eng. 7, 8419-8428. doi: 10.1021/acssuschemeng.9b00056

Wu, R., Tsiakaras, P., and Shen, P. K. (2019b). Facile synthesis of bimetallic Pt-Pd symmetry-broken concave nanocubes and their enhanced activity toward oxygen reduction reaction. Appl. Cataly. B Environ. 251, 49-56. doi: 10.1016/j.apcatb.2019.03.045

$\mathrm{Wu}$, Z. (2012). Anti-galvanic reduction of thiolate-protected gold and silver nanoparticles. Angew. Chem. Int. Ed. Engl. 51, 2934-2938. doi: 10.1002/anie.201107822

Xia, X., Wang, Y., Ruditskiy, A., and Xia, Y. (2013). 25th anniversary article: galvanic replacement: a simple and versatile route to hollow nanostructures with tunable and well-controlled properties. Adv. Mater. 25, 6313-6333. doi: 10.1002/adma.201302820

Xie, S., Lu, N., Xie, Z., Wang, J., Kim, M. J., and Xia, Y. (2012). Synthesis of Pd-Rh core-frame concave nanocubes and their conversion to Rh cubic nanoframes by selective etching of the Pd cores. Angew. Chem. Int. Ed. 51, 10266-10270. doi: 10.1002/anie.201206044

$\mathrm{Xu}$, C., Liu, A., Qiu, H., and Liu, Y. (2011). Nanoporous PdCu alloy with enhanced electrocatalytic performance. Electrochem. Commun. 13, 766-769. doi: 10.1016/j.elecom.2011.04.007
Xu, C., Liu, Y., Zhang, H., and Geng, H. (2013). A nanoporous PdCo alloy as a highly active electrocatalyst for the oxygen-reduction reaction and formic acid electrooxidation. Chem. Asian J. 8, 2721-2728. doi: 10.1002/asia.2013 00607

Xu, Y., Wu, R., Zhang, J., Shi, Y., and Zhang, B. (2013). Anion-exchange synthesis of nanoporous FeP nanosheets as electrocatalysts for hydrogen evolution reaction. Chem. Commun. 49, 6656-6658. doi: 10.1039/c3cc 43107j

Yadav, T. P., Yadav, R. M., and Singh, D. P. (2012). Mechanical milling: a top down approach for the synthesis of nanomaterials and nanocomposites. Nanosci. Nanotechnol. 2, 22-48. doi: 10.5923/j.nn.20120203.01

Yang, F., Zhao, P., Hua, X., Luo, W., Cheng, G., Xing, W., et al. (2016). A cobalt-based hybrid electrocatalyst derived from a carbon nanotube inserted metal-organic framework for efficient water-splitting. J. Mater. Chem. A 4, 16057-16063. doi: 10.1039/x0xx00000x

Ye, W., Guo, X., Xie, F., Zhu, R., Zhao, Q., and Yang, J. (2014). Kinetics-controlled growth of bimetallic RhAg on Au nanorods and their catalytic properties. Nanoscale 6, 4258-4263. doi: 10.1039/c3nr05775e

Ye, W., Kou, S., Guo, X., Xie, F., Sun, H., Lu, H., et al. (2015). Controlled synthesis of bimetallic $\mathrm{Pd}-\mathrm{Rh}$ nanoframes and nanoboxes with high catalytic performances. Nanoscale 7, 9558-9562. doi: 10.1039/c4nr06917j

Ye, X., Zheng, C., Chen, J., Gao, Y., and Murray, C. B. (2013). Using binary surfactant mixtures to simultaneously improve the dimensional tunability and monodispersity in the seeded growth of gold nanorods. Nano Lett. 13, 765-771. doi: $10.1021 / \mathrm{nl} 304478 \mathrm{~h}$

Yuan, X., Dou, X., Zheng, K., and Xie, J. (2015). Recent advances in the synthesis and applications of ultrasmall bimetallic nanoclusters. Part. Part. Syst. Char. 32, 613-629. doi: 10.1002/ppsc.201400212

Zhang, H., Watanabe, T., Okumura, M., Haruta, M., and Toshima, N. (2012). Catalytically highly active top gold atom on palladium nanocluster. Nat. Mater. 11, 49-52. doi: 10.1038/nmat3143

Zhang, J., Wan, L., Liu, L., Deng, Y., Zhong, C., and Hu, W. (2016). PdPt bimetallic nanoparticles enabled by shape control with halide ions and their enhanced catalytic activities. Nanoscale 8, 3962-3972. doi: 10.1039/C5NR05971B

Zhang, L., Xie, Z., and Gong, J. (2016). Shape-controlled synthesis of AuPd bimetallic nanocrystals for catalytic applications. Chem. Soc. Rev. 45, 3916-3934. doi: 10.1039/c5cs00958h

Zhang, W., Yang, J., and Lu, X. (2012). Tailoring galvanic replacement reaction for the preparation of $\mathrm{Pt} / \mathrm{Ag}$ bimetallic hollow nanostructures with controlled number of voids. ACS Nano 6, 7397-7405. doi: 10.1021/nn302590k

Zheng, G., Wang, J., Kong, L., Cheng, H., and Liu, J. (2012). Cellularlike gold nanofeet: synthesis, functionalization, and surface enhanced fluorescence detection for mercury contaminations. Plasmonics 7, 487-494. doi: $10.1007 /$ s11468-012-9333-9

Zheng, Z., Tachikawa, T., and Majima, T. (2014). Single-particle study of Ptmodified Au nanorods for plasmon-enhanced hydrogen generation in visible to near-infrared region. J. Am. Chem. Soc. 136, 6870-6873. doi: 10.1021/ja502704n

Zhu, X., Jia, H., Zhu, X. M., Cheng, S., Zhuo, X., Qin, F., et al. (2017). Selective $\mathrm{Pd}$ deposition on $\mathrm{Au}$ nanobipyramids and $\mathrm{Pd}$ site-dependent plasmonic photocatalytic activity. Adv. Func. Mater. 27:1700016. doi: 10.1002/adfm.201700016

Conflict of Interest: The authors declare that the research was conducted in the absence of any commercial or financial relationships that could be construed as a potential conflict of interest.

Copyright (c) 2020 Bhol, Bhavya, Swain, Saxena and Samal. This is an open-access article distributed under the terms of the Creative Commons Attribution License (CC $B Y)$. The use, distribution or reproduction in other forums is permitted, provided the original author(s) and the copyright owner(s) are credited and that the original publication in this journal is cited, in accordance with accepted academic practice. No use, distribution or reproduction is permitted which does not comply with these terms. 